Pacific

Journal of

Mathematics

SURFACES OF PRESCRIBED MEAN CURVATURE $H(x, y, z)$ WITH ONE-TO-ONE CENTRAL PROJECTION ONTO A PLANE

FRIEDRICH SAUVIGNY 


\title{
SURFACES OF PRESCRIBED MEAN CURVATURE $H(x, y, z)$ WITH ONE-TO-ONE CENTRAL PROJECTION ONTO A PLANE
}

\author{
FRIEDRICH SAUVIGNY
}

\section{Dedicated to the memory of Professor Stefan Hildebrandt in gratitude}

\begin{abstract}
When we consider surfaces of prescribed mean curvature $H$ with a one-toone orthogonal projection onto a plane, we have to study the nonparametric $\boldsymbol{H}$-surface equation. Now the $\boldsymbol{H}$-surfaces with a one-to-one central projection onto a plane lead to an interesting elliptic differential equation, which is derived in Section 2; in the case $H=0$ this PDE was invented by T. Radó. We establish the uniqueness of the Dirichlet problem for this $\boldsymbol{H}$-surface equation in central projection in Section 3, and develop an estimate for the maximal deviation of large $\boldsymbol{H}$-surfaces from their boundary values, resembling an inequality by J. Serrin. In Section 4 we provide a Bernstein-type result for the case $H=0$ and classify the entire solutions of the minimal surface equation in central projection. We also solve the Dirichlet problem for $\boldsymbol{H}=\mathbf{0}$ by a variational method. In Section 5 we solve the Dirichlet problem for nonvanishing $H$ with compact support via a nonlinear continuity method, and we construct large $\boldsymbol{H}$-surfaces bounding extreme contours by an approximation. Finally, in Section 6 we solve the Dirichlet problem on discs for the nonparametric $\boldsymbol{H}$-surface equation in central projection under certain restrictions for the mean curvature.
\end{abstract}

\section{Introduction}

In Plateau's problem for variable $H=H(x, y, z)$, one constructs branched immersions of prescribed mean curvature $H(x, y, z)$ bounding a given Jordan contour $\Gamma$ in $\mathbb{R}^{3}$ by minimizing an energy functional (see [Dierkes et al. 2010a, Part II]). This parametric $H$-surface

$$
X=X(u, v)=(x(u, v), y(u, v), z(u, v))
$$

satisfies Rellich's nonlinear elliptic system (3-31) and is given in conformal parameters - apart from the isolated branch points. In [Sauvigny 1982], this parametric

MSC2010: 35J60, 53A10.

Keywords: surfaces of prescribed mean curvature, solution of the Dirichlet problem, $H$-surface equation in central projection, large $H$-surfaces for Plateau's problem. 
surface $X$ is shown to be a graph $z=\zeta(x, y)$ above the $(x, y)$-plane for certain contours $\Gamma$ and solves the Dirichlet problem for the nonparametric $H$-surface equation to the given boundary values.

In the present paper we solve the Dirichlet problem for $H$-surfaces in the representation (2-3) with a one-to-one central projection by a nonlinear continuity method (compare Theorem 5.1) and an approximation (see Theorem 6.1). We start with a solution of Plateau's problem for $H=0$ which possesses a one-to-one central projection (see Theorem 4.1). Having answered the uniqueness question (compare Theorem 3.1), we study intensively the stability and the compactness of this boundary value problem with the aid of [Sauvigny 1982, Satz 1]. In the minimal surface case, the relevant PDEs (2-17) and (2-24) already appear in a paper by T. Radó [1932] — but the inhomogeneous equations seem to be investigated here for the first time.

We can determine the set of entire solutions for the nonparametric minimal surface equation in central projection (compare Theorem 4.2). While minimal surfaces remain in the convex hull of their bounding contour, this is not the case for surfaces of prescribed mean curvature. However, we can estimate the deviation of our solution from their boundary values by comparison with large spherical caps (see Theorems 2.1 and 3.2). These surfaces do not belong to the family of graphs; however, they possess a one-to-one central projection and can be used here. Moreover, we can construct large solutions of Plateau's problem by a continuity and approximation method (compare Theorem 5.2).

\section{The $H$-surface equation in central projection}

It is well-known that the set of surfaces of constant mean curvature $H \in \mathbb{R}$ is invariant under translations and rotations. When we consider these $H$-surfaces with one-to-one central projection onto a plane $\mathcal{E}$, we can assume by translation that the origin $(0,0,0) \in \mathbb{R}^{3}$ represents the center of projection. Furthermore, we can attain by rotation that this plane $\mathcal{E}$ is parallel to the $x y$-plane. Now $H$-surfaces are transformed into $\left(a^{-1} \cdot H\right)$-surfaces after a dilation by the factor $a \in \mathbb{R} \backslash\{0\}$. Therefore, we can select the set

$$
\mathcal{E}:=\left\{(x, y, 1) \in \mathbb{R}^{3} \mid x, y \in \mathbb{R}\right\}
$$

without loss of generality as our projection plane after a suitable dilation. For the general study of $H$-surfaces with prescribed mean curvature $H=H(x, y, z)$ on domains in the Euclidean space, we refer our readers to Chapter 5 of the treatise [Dierkes et al. 2010a] by U. Dierkes, S. Hildebrandt, and F. Sauvigny.

Choose an arbitrary domain $\Omega \subset \mathbb{R}^{2}$ in the plane; with the real-valued function

$$
\varrho=\varrho(x, y) \in C^{2}(\Omega, \mathbb{R})
$$


we associate the vector-valued function

$$
X=X(x, y):=\varrho(x, y) \cdot(x, y, 1), \quad(x, y) \in \Omega .
$$

At all points $(x, y) \in \Omega$ with $\varrho(x, y) \neq 0$, we obtain in (2-3) a differentialgeometrically regular surface with one-to-one central projection onto the plane $\mathcal{E}$.

In this context, let us calculate the first derivatives of the surface $X$, namely

$$
\begin{aligned}
& X_{x}(x, y)=\varrho_{x}(x, y) \cdot(x, y, 1)+\varrho(x, y) \cdot(1,0,0), \\
& X_{y}(x, y)=\varrho_{y}(x, y) \cdot(x, y, 1)+\varrho(x, y) \cdot(0,1,0),
\end{aligned}
$$

and the coefficients of its first fundamental form, which are

$$
\begin{aligned}
X_{x}^{2}(x, y) & =X_{x} \cdot X_{x}(x, y) \\
& =\varrho_{x}^{2}(x, y) \cdot\left(x^{2}+y^{2}+1\right)+2 \varrho(x, y) x \varrho_{x}(x, y)+\varrho^{2}(x, y), \\
X_{x} \cdot X_{y} & =\varrho_{x}(x, y) \varrho_{y}(x, y)\left(x^{2}+y^{2}+1\right)+\varrho(x, y)\left[y \varrho_{x}(x, y)+x \varrho_{y}(x, y)\right], \\
X_{y}^{2}(x, y) & =\varrho_{y}^{2}(x, y) \cdot\left(x^{2}+y^{2}+1\right)+2 \varrho(x, y) y \varrho_{y}(x, y)+\varrho^{2}(x, y),
\end{aligned}
$$

for $(x, y) \in \Omega$. Furthermore, we determine the exterior product of the vectors (2-4) as follows:

$$
\begin{aligned}
X_{x} & \wedge X_{y}(x, y) \\
& =\left(\varrho_{x} \cdot(x, y, 1)+\varrho \cdot(1,0,0)\right) \wedge\left(\varrho_{y} \cdot(x, y, 1)+\varrho \cdot(0,1,0)\right) \\
& =\varrho \varrho_{x}(x, y) \cdot(-1,0, x)+\varrho \varrho_{y}(x, y) \cdot(0,-1, y)+\varrho^{2}(x, y) \cdot(0,0,1) \\
& =\varrho(x, y) \cdot\left(-\varrho_{x}(x, y),-\varrho_{y}(x, y), \varrho(x, y)+x \varrho_{x}(x, y)+y \varrho_{y}(x, y)\right) .
\end{aligned}
$$

The surface element $W(x, y)$ is given by

$$
\begin{aligned}
W(x, y)^{2}:= & \left|X_{x} \wedge X_{y}(x, y)\right|^{2} \\
= & \varrho^{2}(x, y) \cdot\left(|\nabla \varrho(x, y)|^{2}+\left[\varrho(x, y)+x \varrho_{x}+y \varrho_{y}\right]^{2}\right) \\
= & \varrho^{2}(x, y) \cdot\left(\varrho^{2}(x, y)+\left(1+x^{2}\right) \varrho_{x}^{2}+\left(1+y^{2}\right) \varrho_{y}^{2}\right. \\
& \left.\quad+2 x y \varrho_{x} \varrho_{y}+2 x \varrho \varrho_{x}+2 y \varrho_{y}\right) .
\end{aligned}
$$

Therefore, the equivalence

$$
X_{x} \wedge X_{y}(x, y) \neq 0 \text { if and only if } \varrho(x, y) \neq 0,
$$

which we have already used above, holds true.

We determine the second derivatives of our surface (2-3) via (2-4) and obtain

$$
\begin{aligned}
& X_{x x}(x, y)=\varrho_{x x}(x, y) \cdot(x, y, 1)+2 \varrho_{x}(x, y) \cdot(1,0,0), \\
& X_{x y}(x, y)=\varrho_{x y}(x, y) \cdot(x, y, 1)+\left(\varrho_{y}(x, y), \varrho_{x}(x, y), 0\right), \\
& X_{y y}(x, y)=\varrho_{y y}(x, y) \cdot(x, y, 1)+2 \varrho_{y}(x, y) \cdot(0,1,0) .
\end{aligned}
$$


With the aid of (2-6) and (2-9), we determine the coefficients of its second fundamental form - multiplied by $W(x, y)$ - in the following triple products:

$$
\begin{aligned}
& \left.\left(X_{x}, X_{y}, X_{x x}\right)\right|_{(x, y)}=\varrho^{2} \varrho_{x x}(x, y)-2 \varrho \varrho_{x}^{2}(x, y), \\
& \left.\left(X_{x}, X_{y}, X_{x y}\right)\right|_{(x, y)}=\varrho^{2} \varrho_{x y}(x, y)-2 \varrho \varrho_{x} \varrho_{y}(x, y), \\
& \left.\left(X_{x}, X_{y}, X_{y y}\right)\right|_{(x, y)}=\varrho^{2} \varrho_{y y}(x, y)-2 \varrho \varrho_{y}^{2}(x, y) .
\end{aligned}
$$

For an adequate geometric formulation we need some definitions.

Definition 2.1. With each domain $\Omega \subset \mathbb{R}^{2}$ we associate the cone $(2-11) \mathcal{C}(\Omega):=\left\{(\xi, \eta, \zeta) \in \mathbb{R}^{3} \mid \xi=r x, \eta=r y, \zeta=r,(x, y) \in \Omega, 0<r<+\infty\right\}$, where $\Omega \times\{1\} \subset \mathbb{R}^{3}$ represents its base and $(0,0,0)$ its vertex. The cone $\mathcal{C}(\Omega)$ consists of the generating lines

$$
L_{(x, y)}:=\left\{(r x, r y, r) \in \mathbb{R}^{3} \mid 0<r<+\infty\right\} \text { for all }(x, y) \in \Omega .
$$

The boundary of our cone $\partial \mathcal{C}(\Omega)$ is composed of the generating lines $L_{(x, y)}$, $(x, y) \in \partial \Omega$.

Definition 2.2. At first, we define the logarithmic mean curvature on the cylinder $\Omega \times \mathbb{R}$ by the continuous function

$$
D=D(x, y, z): \Omega \times \mathbb{R} \rightarrow \mathbb{R} \in C^{0}(\Omega \times \mathbb{R}) .
$$

Then we prescribe the associate mean curvature on the cone $\mathcal{C}(\Omega)$ by setting

$$
\begin{gathered}
H(\xi, \eta, \zeta):=\frac{D(x, y, \ln r)}{r}, \\
(\xi, \eta, \zeta)=(r x, r y, r) \in \mathcal{C}(\Omega), \quad(x, y) \in \Omega, \quad 0<r<+\infty .
\end{gathered}
$$

At all points with $\varrho(x, y)>0$, the representation (2-3) yields a surface of prescribed mean curvature $H$ from (2-12) and (2-13), or briefly an $H$-surface, if and only if the following partial differential equation (PDE) holds true:

(2-14) $2 D(x, y, \ln \varrho) \varrho^{2}$

$$
\begin{aligned}
\times & \left(\varrho^{2}+\left(1+x^{2}\right) \varrho_{x}^{2}+\left(1+y^{2}\right) \varrho_{y}^{2}+2 x y \varrho_{x} \varrho_{y}+2 x \varrho \varrho_{x}+2 y \varrho \varrho_{y}\right)^{3 / 2} \\
= & 2 H(\varrho x, \varrho y, \varrho) \varrho^{3} \\
& \times\left(\varrho^{2}+\left(1+x^{2}\right) \varrho_{x}^{2}+\left(1+y^{2}\right) \varrho_{y}^{2}+2 x y \varrho_{x} \varrho_{y}+2 x \varrho \varrho_{x}+2 y \varrho \varrho_{y}\right)^{3 / 2} \\
= & 2 H(\varrho x, \varrho y, \varrho) W^{3}(x, y) \\
= & X_{y}^{2}\left(X_{x}, X_{y}, X_{x x}\right)-2\left(X_{x} \cdot X_{y}\right)\left(X_{x}, X_{y}, X_{x y}\right)+X_{x}^{2}\left(X_{x}, X_{y}, X_{y y}\right) \\
= & \varrho^{2}\left(X_{y}^{2} \varrho_{x x}(x, y)-2\left(X_{x} \cdot X_{y}\right) \varrho_{x y}(x, y)+X_{x}^{2} \varrho_{y y}(x, y)\right) \\
& \quad-2 \varrho(x, y)\left(X_{y}^{2} \varrho_{x}^{2}(x, y)-2\left(X_{x} \cdot X_{y}\right) \varrho_{x} \varrho_{y}(x, y)+X_{x}^{2} \varrho_{y}^{2}(x, y)\right) .
\end{aligned}
$$


Besides the prescription (2-13) for the mean curvature, we have used the identity (2-7) for the surface element and the equations (2-10) for the triple products. With the aid of the relations (2-5) we immediately calculate

$$
X_{y}^{2} \varrho_{x}^{2}(x, y)-2\left(X_{x} \cdot X_{y}\right) \varrho_{x} \varrho_{y}(x, y)+X_{x}^{2} \varrho_{y}^{2}(x, y)=\varrho^{2}(x, y)|\nabla \varrho(x, y)|^{2} .
$$

When we insert the identity (2-15) into the equation (2-14), we arrive at the PDE

(2-16) $2 D(x, y, \ln \varrho)$

$$
\begin{aligned}
& \times\left(\varrho^{2}+\left(1+x^{2}\right) \varrho_{x}^{2}+\left(1+y^{2}\right) \varrho_{y}^{2}+2 x y \varrho_{x} \varrho_{y}+2 x \varrho_{x}+2 y \varrho \varrho_{y}\right)^{3 / 2} \\
& =X_{y}^{2} \varrho_{x x}(x, y)-2\left(X_{x} \cdot X_{y}\right) \varrho_{x y}(x, y)+X_{x}^{2} \varrho_{y y}(x, y) \\
& \quad-2 \varrho(x, y)|\nabla \varrho(x, y)|^{2} .
\end{aligned}
$$

Taking the coefficients of the first fundamental form (2-5) into account, we obtain the PDE

(2-17) $2 D(x, y, \ln \varrho)$

$$
\begin{aligned}
\times & \left(\varrho^{2}+\left(1+x^{2}\right) \varrho_{x}^{2}+\left(1+y^{2}\right) \varrho_{y}^{2}+2 x y \varrho_{x} \varrho_{y}+2 x \varrho_{x}+2 y \varrho \varrho_{y}\right)^{3 / 2} \\
= & \left(\varrho_{y}^{2} \cdot\left(x^{2}+y^{2}+1\right)+2 \varrho y \varrho_{y}+\varrho^{2}\right) \varrho_{x x} \\
& \quad-2\left(\varrho_{x} \varrho_{y} \cdot\left(x^{2}+y^{2}+1\right)+\varrho\left[y \varrho_{x}+x \varrho_{y}\right]\right) \varrho_{x y} \\
& +\left(\varrho_{x}^{2} \cdot\left(x^{2}+y^{2}+1\right)+2 \varrho x \varrho_{x}+\varrho^{2}\right) \varrho_{y y}(x, y)-2 \varrho|\nabla \varrho(x, y)|^{2},
\end{aligned}
$$

for $(x, y) \in \Omega$.

Since our surface $X$ is regular in $\Omega$, we can assume the property

$$
\varrho(x, y)>0, \quad(x, y) \in \Omega,
$$

after an eventual reflection. Now we use the logarithmic representation

$$
\sigma(x, y):=\ln \varrho(x, y), \quad(x, y) \in \Omega .
$$

Then we determine their first derivatives

$$
\sigma_{x}(x, y)=\frac{\varrho_{x}}{\varrho}(x, y), \quad \sigma_{y}(x, y)=\frac{\varrho_{y}}{\varrho}(x, y), \quad(x, y) \in \Omega,
$$

as well as their second derivatives

$$
\begin{aligned}
\left.\left(\sigma_{x x}+\sigma_{x}^{2}\right)\right|_{(x, y)} & =\frac{\varrho_{x x}}{\varrho}(x, y), \\
\left.\left(\sigma_{x y}+\sigma_{x} \sigma_{y}\right)\right|_{(x, y)} & =\frac{\varrho_{x y}}{\varrho}(x, y), \\
\left.\left(\sigma_{y y}+\sigma_{y}^{2}\right)\right|_{(x, y)} & =\frac{\varrho_{y y}}{\varrho}(x, y) .
\end{aligned}
$$


From the identity (2-15) we deduce the relation

$$
\begin{aligned}
X_{y}^{2} \sigma_{x}^{2}(x, y)-2\left(X_{x} \cdot X_{y}\right) \sigma_{x} \sigma_{y}(x, y)+X_{x}^{2} \sigma_{y}^{2}(x, y) & =|\nabla \varrho(x, y)|^{2} \\
& =\varrho^{2}|\nabla \sigma(x, y)|^{2} .
\end{aligned}
$$

Into the equation (2-17) we insert the second derivatives (2-21) and observe (2-22) to obtain

$$
\begin{aligned}
2 D(x, y, \ln \varrho) \varrho^{2} & \\
\times(1+ & \left.\left(1+x^{2}\right)\left(\frac{\varrho_{x}}{\varrho}\right)^{2}+\left(1+y^{2}\right)\left(\frac{\varrho_{y}}{\varrho}\right)^{2}+2 x y \frac{\varrho_{x}}{\varrho} \frac{\varrho_{y}}{\varrho}+2 x \frac{\varrho_{x}}{\varrho}+2 y \frac{\varrho_{y}}{\varrho}\right)^{3 / 2} \\
= & \left(\varrho_{y}^{2} \cdot\left(x^{2}+y^{2}+1\right)+2 \varrho y \varrho_{y}+\varrho^{2}\right) \frac{\varrho_{x x}}{\varrho} \\
& \quad-2\left(\varrho_{x} \varrho_{y} \cdot\left(x^{2}+y^{2}+1\right)+\varrho\left[y \varrho_{x}+x \varrho_{y}\right]\right) \frac{\varrho_{x y}}{\varrho} \\
& +\left(\varrho_{x}^{2} \cdot\left(x^{2}+y^{2}+1\right)+2 \varrho x \varrho_{x}+\varrho^{2}\right) \frac{\varrho_{y y}}{\varrho}(x, y) \\
& -2|\nabla \varrho(x, y)|^{2} \\
= & \left(\varrho_{y}^{2} \cdot\left(x^{2}+y^{2}+1\right)+2 \varrho y \varrho_{y}+\varrho^{2}\right) \sigma_{x x} \\
& -2\left(\varrho_{x} \varrho_{y} \cdot\left(x^{2}+y^{2}+1\right)+\varrho\left[y \varrho_{x}+x \varrho_{y}\right]\right) \sigma_{x y} \\
& +\left(\varrho_{x}^{2} \cdot\left(x^{2}+y^{2}+1\right)+2 \varrho x \varrho_{x}+\varrho^{2}\right) \sigma_{y y}(x, y) \\
& -\varrho^{2}(x, y)|\nabla \sigma(x, y)|^{2},
\end{aligned}
$$

for $(x, y) \in \Omega$. Now we use formulae (2-20) for the first derivatives and arrive at the PDE

$$
\begin{aligned}
2 D(x, y, \sigma) & \left(1+\left(1+x^{2}\right) \sigma_{x}^{2}+\left(1+y^{2}\right) \sigma_{y}^{2}+2 x y \sigma_{x} \sigma_{y}+2 x \sigma_{x}+2 y \sigma_{y}\right)^{3 / 2} \\
= & \left(\sigma_{y}^{2} \cdot\left(x^{2}+y^{2}+1\right)+2 y \sigma_{y}+1\right) \sigma_{x x} \\
& -2\left(\sigma_{x} \sigma_{y} \cdot\left(x^{2}+y^{2}+1\right)+\left[y \sigma_{x}+x \sigma_{y}\right]\right) \sigma_{x y} \\
& +\left(\sigma_{x}^{2} \cdot\left(x^{2}+y^{2}+1\right)+2 x \sigma_{x}+1\right) \sigma_{y y}(x, y)-|\nabla \sigma(x, y)|^{2}
\end{aligned}
$$

for $(x, y) \in \Omega$.

Definition 2.3. Let us address the PDE (2-17) as the $H$-surface equation in central projection and the PDE (2-24) as the logarithmic H-surface equation. In the special case $D \equiv 0 \equiv H$, we speak of the PDE (2-17) as the minimal surface equation in central projection and of the PDE (2-24) as the logarithmic minimal surface equation.

In the case $D=D(x, y): \Omega \rightarrow \mathbb{R}$, where the logarithmic mean curvature does not depend on the $z$-variable, we prescribe the associate mean curvature $H$ from (2-13) on the base of the cone $\mathcal{C}(\Omega)$. The mean curvature is positive-homogeneously 
continued on each generating line $L_{(x, y)},(x, y) \in \Omega$, of the degree -1 . Then our PDE (2-17) is positive-homogeneous in the following sense: for any positive solution $\varrho(x, y)$ of (2-17) and all parameters $a>0$, the function $a \cdot \varrho(x, y)$ solves this differential equation as well. In the special case that the logarithmic mean curvature

$$
\widehat{D}(x, y):=\frac{-2}{1+x^{2}+y^{2}}, \quad(x, y) \in \mathbb{R}^{2},
$$

is prescribed on the base of our cone, we can explicitly solve the PDE (2-17) as follows.

Theorem 2.1. Let the right-hand side $\widehat{D}$ from (2-25) with its homogeneous continuation $\widehat{H}$ of (2-13) be given on the cone $\mathcal{C}(\Omega)$ for $\Omega=\mathbb{R}^{2}$. Then the functions

(2-26) $\hat{\varrho}(x, y):=\frac{a}{1+x^{2}+y^{2}}, \quad(x, y) \in \mathbb{R}^{2}, \quad$ for arbitrary parameters $a>0$, solve the $\widehat{H}$-surface equation (2-17) in central projection.

Proof. Equivalently to the PDE (2-17) for the function $\hat{\varrho}$, we consider the PDE (2-24) for its logarithmic representation $\hat{\sigma}(x, y):=\ln \hat{\varrho}(x, y),(x, y) \in \mathbb{R}^{2}$, and obtain

$$
\begin{gathered}
\hat{\sigma}(x, y)=\ln a-\ln \left(1+x^{2}+y^{2}\right), \\
\hat{\sigma}_{x}(x, y)=\frac{-2 x}{1+x^{2}+y^{2}}, \quad \hat{\sigma}_{y}(x, y)=\frac{-2 y}{1+x^{2}+y^{2}} .
\end{gathered}
$$

We easily determine the expressions

$$
\begin{aligned}
\left(1+x^{2}\right) \hat{\sigma}_{x}^{2}+\left(1+y^{2}\right) \hat{\sigma}_{y}^{2}+2 x y \hat{\sigma}_{x} \hat{\sigma}_{y}+2 x \hat{\sigma}_{x}+2 y \hat{\sigma}_{y} & =0, \\
\hat{\sigma}_{y}^{2} \cdot\left(x^{2}+y^{2}+1\right)+2 y \hat{\sigma}_{y}+1 & =1, \\
\hat{\sigma}_{x} \hat{\sigma}_{y} \cdot\left(x^{2}+y^{2}+1\right)+\left[y \hat{\sigma}_{x}+x \hat{\sigma}_{y}\right] & =0, \\
\hat{\sigma}_{x}^{2} \cdot\left(x^{2}+y^{2}+1\right)+2 x \hat{\sigma}_{x}+1 & =1,
\end{aligned}
$$

for $(x, y) \in \Omega$. Therefore, the PDE (2-24) is reduced to the equation

$$
\begin{aligned}
\Delta \hat{\sigma}(x, y)-|\nabla \hat{\sigma}(x, y)|^{2} & =\frac{-4}{1+x^{2}+y^{2}}+\frac{4 x^{2}+4 y^{2}}{\left(1+x^{2}+y^{2}\right)^{2}}-\frac{4 x^{2}+4 y^{2}}{\left(1+x^{2}+y^{2}\right)^{2}} \\
& =\frac{-4}{1+x^{2}+y^{2}}=2 \widehat{D}(x, y),
\end{aligned}
$$

for $(x, y) \in \mathbb{R}^{2}$. Consequently, the PDE (2-17) with the right-hand side from (2-25) is satisfied for the functions (2-26).

Remark. For each $a>0$, the surface (2-3) on the domain $\Omega=\mathbb{R}^{2}$ with the function (2-26) represents a surface of constant mean curvature $-2 / a$ with one-to-one central projection onto the plane $\mathcal{E}$. More precisely, we obtain a sphere of radius $a / 2$ about 
the center $(0,0, a / 2)$, where its south pole $(0,0,0)$ has been exempted. We shall use these solutions, which represent a foliation of spheres, as comparison surfaces in the next section.

Proof. In the case $a=1$, the equations (2-3) and (2-26) represent the stereographic projection of this sphere onto the plane $\mathcal{E}$. Here we employ a theorem of Euclid on right triangles: the square of a small side equals its projection on the hypotenuse times the hypotenuse. Based on a simple diagram with a right triangle, we thus obtain $1^{2}=\varrho(x, y) \sqrt{1+x^{2}+y^{2}} \cdot \sqrt{1+x^{2}+y^{2}}$, which yields

$$
\varrho(x, y)=\frac{1}{1+x^{2}+y^{2}} .
$$

By a dilation with the factor $a$, we can easily inspect the general case $a>0$.

\section{Uniqueness of Dirichlet's problem and estimates}

Definition 3.1. Let the logarithmic mean curvature $D(x, y, z): \Omega \times \mathbb{R} \rightarrow \mathbb{R}$ be given on the cylinder adjoint to the bounded Jordan domain $\Omega \subset \mathbb{R}^{2}$, with its associate mean curvature $H(\xi, \eta, \zeta): \mathcal{C}(\Omega) \rightarrow \mathbb{R}$ from $(2-13)$ on the cone $\mathcal{C}(\Omega)$. On the Jordan contour $\partial \Omega$ let the positive continuous boundary distribution $\phi: \partial \Omega \rightarrow(0,+\infty)$ be prescribed. Then the positive solution

$$
\varrho=\varrho(x, y): \bar{\Omega} \rightarrow(0,+\infty) \in C^{2}(\Omega) \cap C^{0}(\bar{\Omega})
$$

of the PDE (2-17) under the Dirichlet boundary condition

$$
\varrho(x, y)=\phi(x, y) \quad \text { for all }(x, y) \in \partial \Omega
$$

is named the solution of the Dirichlet problem $\boldsymbol{P}(\Omega, \phi, H)$ for the $H$-surface equation in central projection.

Definition 3.2. The logarithmic mean curvature $D(x, y, z): \Omega \times \mathbb{R} \rightarrow \mathbb{R}$ satisfies the monotonicity condition if, for each point $(x, y)$ in the domain $\Omega$, the function $d$ defined by

$$
d(z):=D(x, y, z) \quad \text { for } z \in \mathbb{R}
$$

is of class $C^{1}$ and satisfies

$$
d^{\prime}(z)=\frac{\partial}{\partial z} D(x, y, z) \geq 0 \quad \text { for } z \in \mathbb{R} .
$$

The maximum principle for elliptic equations implies the following.

Theorem 3.1 (uniqueness of $\boldsymbol{P}(\Omega, \phi, H))$. Let $\varrho^{(j)}=\varrho^{(j)}(x, y), j=1,2$, denote two solutions of the Dirichlet problem $\boldsymbol{P}(\Omega, \phi, H)$ in the Jordan domain $\Omega \subset \mathbb{R}^{2}$, where the logarithmic mean curvature satisfies the monotonicity condition. Then

$$
\varrho^{(1)}(x, y)=\varrho^{(2)}(x, y) \quad \text { for }(x, y) \in \bar{\Omega} .
$$


Proof. Let us consider two solutions

$$
\varrho^{(j)}=\varrho^{(j)}(x, y): \mathbb{R}^{2} \rightarrow(0,+\infty) \in C^{2}(\Omega) \cap C^{0}(\bar{\Omega}), \quad \text { with } j=1,2,
$$

of the Dirichlet problem $\boldsymbol{P}(\Omega, \phi, H)$. We can apply the maximum principle to the difference of their logarithmic representations,

$$
\sigma^{(j)}(x, y):=\ln \varrho^{(j)}(x, y), \quad(x, y) \in \bar{\Omega}, \quad \text { with } j=1,2,
$$

since the associate PDE (2-24) is quasilinear. The elliptic differential operator for the difference function possesses a nonpositive coefficient of 0 order, due to the monotonicity condition (compare [Sauvigny 2012a, Chapter 6, §2]). Therefore, we obtain

$$
\varrho^{(1)}(x, y)=\varrho^{(2)}(x, y), \quad(x, y) \in \bar{\Omega},
$$

and the Dirichlet problem $\boldsymbol{P}(\Omega, \phi, H)$ is uniquely determined.

Furthermore, we prove the following interesting theorem.

Theorem 3.2 (geometric maximum principle). Let $\varrho=\varrho(x, y)$ denote a solution of the Dirichlet problem $\boldsymbol{P}(\Omega, \phi, H)$ in the Jordan domain $\Omega \subset \mathbb{R}^{2}$, which is contained in the disc $\Omega_{b}:=\left\{(x, y) \in \mathbb{R}^{2} \mid x^{2}+y^{2}<b^{2}\right\}$ of radius $0<b<+\infty$. Furthermore, let the logarithmic mean curvature satisfy the monotonicity condition and the inequalities

$$
\widehat{D}(x, y) \leq D(x, y, z) \leq 0 \quad \text { for all }(x, y, z) \in \Omega \times \mathbb{R} .
$$

Then we have the estimate

$$
0<\min _{(\xi, \eta) \in \partial \Omega} \phi(\xi, \eta) \leq \varrho(x, y) \leq\left(1+b^{2}\right) \cdot \max _{(\xi, \eta) \in \partial \Omega} \phi(\xi, \eta),
$$

for all points $(x, y) \in \bar{\Omega}$.

Proof. (1) From (2-17) and (3-4) we infer the elliptic differential inequality

$$
\begin{aligned}
& \left(\varrho_{y}^{2} \cdot\left(x^{2}+y^{2}+1\right)+2 \varrho y \varrho_{y}+\varrho^{2}\right) \varrho_{x x} \\
& \quad-2\left(\varrho_{x} \varrho_{y} \cdot\left(x^{2}+y^{2}+1\right)+\varrho\left[y \varrho_{x}+x \varrho_{y}\right]\right) \varrho_{x y} \\
& \quad+\left(\varrho_{x}^{2} \cdot\left(x^{2}+y^{2}+1\right)+2 \varrho x \varrho_{x}+\varrho^{2}\right) \varrho_{y y}(x, y)-2 \varrho|\nabla \varrho(x, y)|^{2} \leq 0,
\end{aligned}
$$

for $(x, y) \in \Omega$. Within the domain $\Omega$ our function $\varrho$ cannot attain a strict minimum, and the estimate on the left-hand side of (3-5) is established.

(2) We compare the solution $\varrho$ with the spherical solution of Theorem 2.1,

$$
\hat{\varrho}(x, y):=\frac{a}{1+x^{2}+y^{2}}, \quad(x, y) \in \mathbb{R}^{2}, \quad \text { where } a:=\left(1+b^{2}\right) \max _{(\xi, \eta) \in \partial \Omega} \phi(\xi, \eta) .
$$

By construction we have the inequality

$$
\hat{\varrho}(x, y) \geq \varrho(x, y) \quad \text { for all }(x, y) \in \partial \Omega
$$


on the boundary. From the condition (3-4) and the PDE (2-24) for the logarithmic representation

$$
\hat{\sigma}(x, y):=\ln \hat{\varrho}(x, y), \quad(x, y) \in \bar{\Omega},
$$

we deduce the differential inequality

$$
\begin{aligned}
\left(\hat{\sigma}_{y}^{2} \cdot\left(x^{2}+y^{2}+1\right)+2 y \hat{\sigma}_{y}+1\right) \hat{\sigma}_{x x}-2\left(\hat{\sigma}_{x} \hat{\sigma}_{y} \cdot\left(x^{2}+y^{2}+1\right)+\left[y \hat{\sigma}_{x}+x \hat{\sigma}_{y}\right]\right) \hat{\sigma}_{x y} \\
+\left(\hat{\sigma}_{x}^{2} \cdot\left(x^{2}+y^{2}+1\right)+2 x \hat{\sigma}_{x}+1\right) \hat{\sigma}_{y y}(x, y)-|\nabla \hat{\sigma}(x, y)|^{2} \\
=2 \widehat{D}(x, y)\left(1+\left(1+x^{2}\right) \hat{\sigma}_{x}^{2}+\left(1+y^{2}\right) \hat{\sigma}_{y}^{2}+2 x y \hat{\sigma}_{x} \hat{\sigma}_{y}+2 x \hat{\sigma}_{x}+2 y \hat{\sigma}_{y}\right)^{3 / 2} \\
\leq 2 D(x, y, \hat{\sigma}) \\
\quad \quad \times\left(1+\left(1+x^{2}\right) \hat{\sigma}_{x}^{2}+\left(1+y^{2}\right) \hat{\sigma}_{y}^{2}+2 x y \hat{\sigma}_{x} \hat{\sigma}_{y}+2 x \hat{\sigma}_{x}+2 y \hat{\sigma}_{y}\right)^{3 / 2},
\end{aligned}
$$

at all points $(x, y) \in \Omega$. The logarithmic representation

$$
\sigma(x, y):=\ln \varrho(x, y), \quad(x, y) \in \bar{\Omega},
$$

of the function $\varrho$ satisfies the quasilinear PDE (2-24). Together with (3-9) the difference function

$$
\tau(x, y):=\sigma(x, y)-\hat{\sigma}(x, y), \quad(x, y) \in \bar{\Omega},
$$

is subject to the differential inequality

$$
\mathcal{L} \tau(x, y) \geq 0, \quad(x, y) \in \Omega,
$$

for an elliptic differential operator $\mathcal{L}$ (see [Sauvigny 2012a, Chapter 6, §2]). Due to the monotonicity condition, the coefficient for the 0 -order term of $\mathcal{L}$ is nonpositive. Because of (3-8) we have $\tau(x, y) \leq 0$ for $(x, y) \in \partial \Omega$ and hence, by the maximum principle for elliptic operators, $\tau(x, y) \leq 0$ for $(x, y) \in \bar{\Omega}$. With the aid of (3-7) we arrive at the estimate

$$
\varrho(x, y) \leq \hat{\varrho}(x, y) \leq a=\left(1+b^{2}\right) \cdot \max _{(\xi, \eta) \in \partial \Omega} \phi(\xi, \eta), \quad(x, y) \in \bar{\Omega} .
$$

This shows the right-hand side of the statement (3-5) above.

Remark. For embedded surfaces of constant mean curvature, J. Serrin [1969] established a maximum-estimate by the bounding contour; there the reflection method of A. D. Alexandroff was employed. Our method above is based on a foliation of $H$-surfaces with variable mean curvature.

Already at the beginning of the last century, A. Korn and C. H. Müntz solved the boundary value problem of the minimal surface equation for contours deviating only a little from planar curves. From $\S \S 413-415$ of J. C. C. Nitsche's treatise [1975] we learn that Plateau's problem for parametric minimal surfaces with positive second variation is stable with respect to small perturbations of the bounding contour. 
The approximate solutions are obtained by solving a peculiar nonlinear elliptic PDE which is accessible to Banach's fixed point theorem within the Hölder space $C^{2+\alpha}(\bar{B})$ (compare [Dierkes et al. 2010a, §5.6, Proposition 1]). We have used this method in Proposition 1.8 of [Sauvigny 2012b, Chapter 13], in order to establish the stability of the nonparametric $H$-surface equation under small perturbations with respect to the $C^{2+\alpha}$-norm of the boundary data. Since the logarithmic $H$-surface equation (2-24) has a similar structure, we can prove the stability for our Dirichlet problem $\boldsymbol{P}(\Omega, \phi, H)$ under homothetic transformations — near the identity — of the boundary values.

Lemma 3.1 (perturbation result). Let $\Omega \subset \mathbb{R}^{2}$ denote a convex $C^{2+\alpha}$-Jordan domain with $0<\alpha<1$, such that the logarithmic mean curvature

$$
D=D(x, y, z) \in C^{1+\alpha}(\bar{\Omega} \times \mathbb{R}, \mathbb{R})
$$

satisfies the monotonicity condition. For the associate mean curvature $H$ due to (2-13) on the cone $\mathcal{C}(\Omega)$ and the positive boundary distribution $\phi: \partial \Omega \rightarrow(0,+\infty)$ of class $C^{2+\alpha}$, the problem $\boldsymbol{P}(\Omega, \phi, H)$ possesses a solution. Then the Dirichlet problem $\boldsymbol{P}(\Omega, \lambda \phi, H)$ is solvable for all parameters $1-\varepsilon \leq \lambda \leq 1+\varepsilon$, where the quantity $\varepsilon>0$ is sufficiently small.

Proof. (1) Instead of the PDE (2-17) we use the equivalent equation (2-24) and start with a solution $\sigma=\sigma(x, y) \in C^{2+\alpha}(\bar{\Omega})$ of this logarithmic $H$-surface equation. Then we consider the perturbation

$$
\sigma(x, y)+t+\tau(x, y), \quad(x, y) \in \bar{\Omega},
$$

with a parameter $-\epsilon \leq t \leq \epsilon$ and a function $\tau$ in the Banach space

$$
\mathcal{B}:=\left\{\tau=\tau(x, y) \in C^{2+\alpha}(\bar{\Omega}) \mid \tau(x, y)=0 \text { for all }(x, y) \in \partial \Omega\right\} .
$$

We insert (3-11) into (2-24) and observe that our perturbed function (3-11) satisfies the logarithmic $H$-surface equation (2-24) if and only if the function $\tau \in \mathcal{B}$ fulfills the PDE

$$
\begin{aligned}
& \left(\left(\sigma_{y}+\tau_{y}\right)^{2} \cdot\left(x^{2}+y^{2}+1\right)+2 y\left(\sigma_{y}+\tau_{y}\right)+1\right)\left(\sigma_{x x}+\tau_{x x}\right) \\
& -2\left(\left(\sigma_{x}+\tau_{x}\right)\left(\sigma_{y}+\tau_{y}\right) \cdot\left(x^{2}+y^{2}+1\right)+\left[y\left(\sigma_{x}+\tau_{x}\right)+x\left(\sigma_{y}+\tau_{y}\right)\right]\right)\left(\sigma_{x y}+\tau_{x y}\right) \\
& +\left(\left(\sigma_{x}+\tau_{x}\right)^{2} \cdot\left(x^{2}+y^{2}+1\right)+2 x\left(\sigma_{x}+\tau_{x}\right)+1\right)\left(\sigma_{y y}+\tau_{y y}\right) \\
& -\left.|(\nabla \sigma+\nabla \tau)|_{(x, y)}\right|^{2} \\
& =2 D(x, y, \sigma+t+\tau)\left(1+\left(1+x^{2}\right)\left(\sigma_{x}+\tau_{x}\right)^{2}+\left(1+y^{2}\right)\left(\sigma_{y}+\tau_{y}\right)^{2}\right. \\
& \left.\quad+2 x y\left(\sigma_{x}+\tau_{x}\right)\left(\sigma_{y}+\tau_{y}\right)+2 x\left(\sigma_{x}+\tau_{x}\right)+2 y\left(\sigma_{y}+\tau_{y}\right)\right)^{3 / 2},
\end{aligned}
$$

for $(x, y) \in \Omega$. 
(2) On the left-hand side of (3-12) we collect all those terms, where the factors of the set

$$
\mathcal{F}:=\left\{\tau_{x}, \tau_{y}, \tau_{x x}, \tau_{x y}, \tau_{y y}\right\}
$$

appear in the same order, to the following differential operators: the terms of order 0 result in the expression

(3-13) $2 D(x, y, \sigma)\left(1+\left(1+x^{2}\right) \sigma_{x}^{2}+\left(1+y^{2}\right) \sigma_{y}^{2}+2 x y \sigma_{x} \sigma_{y}+2 x \sigma_{x}+2 y \sigma_{y}\right)^{3 / 2}$,

since $\sigma$ solves the PDE (2-24). Then we collect all terms of order 1 to the linear elliptic differential operator

$$
\begin{aligned}
\mathcal{L}_{0} \tau:=( & \left.\sigma_{y}^{2} \cdot\left(x^{2}+y^{2}+1\right)+2 y \sigma_{y}+1\right) \tau_{x x} \\
& -2\left(\sigma_{x} \sigma_{y} \cdot\left(x^{2}+y^{2}+1\right)+\left[y \sigma_{x}+x \sigma_{y}\right]\right) \tau_{x y} \\
& +\left(\sigma_{x}^{2} \cdot\left(x^{2}+y^{2}+1\right)+2 x \sigma_{x}+1\right) \tau_{y y}(x, y) \\
& +a(x, y) \tau_{x}+b(x, y) \tau_{y}
\end{aligned}
$$

with coefficients $a(x, y), b(x, y)$ of the class $C^{\alpha}(\bar{\Omega})$ depending on the solution $\sigma$. The remaining terms of order 2 and 3 are assembled to the nonlinear operator $\mathcal{Q}=\mathcal{Q}(\tau): \mathcal{B} \rightarrow C^{\alpha}(\bar{\Omega})$. On the ball $\mathcal{B}_{\delta}:=\left\{\tau=\tau(x, y) \in \mathcal{B} \mid\|\tau\|_{C^{2+\alpha}(\bar{\Omega})} \leq \delta\right\}$ of radius $\delta>0$ the estimates

$$
\begin{gathered}
\|\mathcal{Q}(\tau)\|_{C^{\alpha}(\bar{\Omega})} \leq L_{1}(\delta)\|\tau\|_{C^{2+\alpha}(\bar{\Omega})} \quad \text { for all } \tau \in \mathcal{B}_{\delta}, \\
\|\mathcal{Q}(\tilde{\tau})-\mathcal{Q}(\hat{\tau})\|_{C^{\alpha}(\bar{\Omega})} \leq L_{2}(\delta)\|\tilde{\tau}-\hat{\tau}\|_{C^{2+\alpha}(\bar{\Omega})} \quad \text { for all } \tilde{\tau}, \hat{\tau} \in \mathcal{B}_{\delta}
\end{gathered}
$$

hold true. Here as well as in (3-18), (3-19), (3-26) below, the constants $L_{j}(\delta)>0$ satisfy $\lim _{\delta \rightarrow 0+} L_{j}(\delta)=0$ for $j=1,2,3,4$. When we respect that all terms in $\mathcal{Q}$ are either quadratic or cubic in $\mathcal{F}$ and control their Hölder-norms, we immediately see the assertions (3-15) above, where the upper inequality implies that the operator $\mathcal{Q}$ is superlinear.

Now the equation (3-12) appears in the equivalent form

(3-16) $\quad \mathcal{L}_{0} \tau+\mathcal{Q}(\tau)$

$$
\begin{gathered}
=2 D(x, y, \sigma+t+\tau)\left(1+\left(1+x^{2}\right)\left(\sigma_{x}+\tau_{x}\right)^{2}+\left(1+y^{2}\right)\left(\sigma_{y}+\tau_{y}\right)^{2}\right. \\
\left.+2 x y\left(\sigma_{x}+\tau_{x}\right)\left(\sigma_{y}+\tau_{y}\right)+2 x\left(\sigma_{x}+\tau_{x}\right)+2 y\left(\sigma_{y}+\tau_{y}\right)\right)^{3 / 2} \\
-2 D(x, y, \sigma)\left(1+\left(1+x^{2}\right) \sigma_{x}^{2}+\left(1+y^{2}\right) \sigma_{y}^{2}\right. \\
\left.+2 x y \sigma_{x} \sigma_{y}+2 x \sigma_{x}+2 y \sigma_{y}\right)^{3 / 2} .
\end{gathered}
$$

(3) We introduce the nonlinear operator

$$
\begin{aligned}
\mathcal{N}(\tau):=(1+ & \left(1+x^{2}\right)\left(\sigma_{x}+\tau_{x}\right)^{2}+\left(1+y^{2}\right)\left(\sigma_{y}+\tau_{y}\right)^{2} \\
& \left.+2 x y\left(\sigma_{x}+\tau_{x}\right)\left(\sigma_{y}+\tau_{y}\right)+2 x\left(\sigma_{x}+\tau_{x}\right)+2 y\left(\sigma_{y}+\tau_{y}\right)\right)^{3 / 2}
\end{aligned}
$$


for $\tau \in \mathcal{B}$, where the power $3 / 2$ is larger than 1 . Therefore, we obtain the estimate

$$
\|\mathcal{N}(\tilde{\tau})-\mathcal{N}(\hat{\tau})\|_{C^{\alpha}(\bar{\Omega})} \leq L_{3}(\delta)\|\tilde{\tau}-\hat{\tau}\|_{C^{2+\alpha}(\bar{\Omega})} \quad \text { for all } \tilde{\tau}, \hat{\tau} \in \mathcal{B}_{\delta} .
$$

Thus we receive the superlinear operator

$$
\begin{gathered}
\mathcal{R}(\tau):=\mathcal{N}(\tau)-\mathcal{N}(0), \quad \tau \in \mathcal{B}, \\
\text { satisfying }\|\mathcal{R}(\tau)\|_{C^{\alpha}(\bar{\Omega})} \leq L_{3}(\delta)\|\tau\|_{C^{2+\alpha}(\bar{\Omega})}, \quad \tau \in \mathcal{B}_{\delta} .
\end{gathered}
$$

Now we rewrite (3-16) into the equivalent form

$$
\begin{aligned}
\mathcal{L}_{0} \tau+\mathcal{Q}(\tau)= & 2 D(x, y, \sigma+t+\tau) \mathcal{N}(\tau)-2 D(x, y, \sigma) \mathcal{N}(0) \\
= & 2 D(x, y, \sigma+t+\tau)(\mathcal{N}(0)+\mathcal{R}(\tau))-2 D(x, y, \sigma) \mathcal{N}(0) \\
= & 2(D(x, y, \sigma+t+\tau)-D(x, y, \sigma)) \mathcal{N}(0) \\
& \quad+2 D(x, y, \sigma+t+\tau) \mathcal{R}(\tau) .
\end{aligned}
$$

(4) Let us determine

$$
\begin{aligned}
& D(x, y, \sigma(x, y)+t+\tau(x, y))-D(x, y, \sigma(x, y)) \\
& \quad=\int_{0}^{1} \frac{d}{d s} D(x, y, s[\tau(x, y)+t]+\sigma(x, y)) d s=c_{0}(x, y)[\tau(x, y)+t],
\end{aligned}
$$

for $(x, y) \in \Omega$, with the nonnegative function

$$
c_{0}(x, y):=\int_{0}^{1} D_{z}(x, y, s[\tau(x, y)+t]+\sigma(x, y)) d s,
$$

due to the monotonicity condition. We insert (3-21) into the PDE (3-20) and arrive at

$$
\mathcal{L}_{0} \tau+\mathcal{Q}(\tau)=2 c_{0}(x, y)[\tau(x, y)+t] \mathcal{N}(0)+2 D(x, y, \sigma+t+\tau) \mathcal{R}(\tau) .
$$

Introducing the coefficient function $c(x, y):=-2 c_{0}(x, y) \mathcal{N}(0) \leq 0,(x, y) \in \Omega$, and the linear elliptic operator $\mathcal{L} \tau:=\mathcal{L}_{0} \tau+c(x, y) \tau, \tau \in \mathcal{B}$, we obtain the PDE

$$
\mathcal{L} \tau=2 t c_{0}(x, y) \mathcal{N}(0)-\mathcal{Q}(\tau)+2 D(x, y, \sigma+t+\tau) \mathcal{R}(\tau)=: \mathcal{M}_{t}(\tau)
$$

with the nonlinear operator $\mathcal{M}_{t}: \mathcal{B} \rightarrow C^{\alpha}(\bar{\Omega})$ on the right-hand side.

For each $\theta>0$, we can find quantities $\delta=\delta(\theta)>0$ and $\varepsilon=\varepsilon(\theta)>0$ such that

$$
\left\|\mathcal{M}_{t}(\tau)\right\|_{C^{\alpha}(\bar{\Omega})} \leq \theta^{-1} \delta \text { for all } \tau \in \mathcal{B}_{\delta} \text { and all }-\varepsilon \leq t \leq+\varepsilon .
$$

This follows from the structure of the operator $\mathcal{M}_{t}$ in (3-23), since the operators $\mathcal{Q}$ and $\mathcal{R}$ are superlinear as in (3-15) and (3-19) and the expression

$$
\sup _{\tau \in \mathcal{B}_{\delta}, t \in[-1,+1]}\|D(x, y, \sigma+t+\tau)\|_{C^{\alpha}(\bar{\Omega})}
$$

is finite, due to the regularity of $D$ on the convex domain $\Omega$. 
Furthermore, with $\delta=\delta(\theta)>0$ and $\varepsilon=\varepsilon(\theta)>0$ we realize the estimate

$$
\begin{aligned}
& \left\|\mathcal{M}_{t}(\tilde{\tau})-\mathcal{M}_{t}(\hat{\tau})\right\|_{C^{\alpha}(\bar{\Omega})} \leq \frac{1}{2 \theta}\|\tilde{\tau}-\hat{\tau}\|_{C^{2+\alpha}(\bar{\Omega})} \\
& \qquad \text { for all } \tilde{\tau}, \hat{\tau} \in \mathcal{B}_{\delta} \text { and }-\varepsilon \leq t \leq+\varepsilon .
\end{aligned}
$$

Here the structure of the operator $\mathcal{M}_{t}$ in (3-23) is combined with the inequalities (3-15), (3-18) and the following estimate, which is based on the regularity of $D$ in the convex domain $\Omega$ :

$$
\begin{array}{r}
\|D(\cdot, \cdot, \sigma+t+\tilde{\tau})-D(\cdot, \cdot, \sigma+t+\hat{\tau})\|_{C^{\alpha}(\bar{\Omega})} \leq L_{4}(\delta)\|\tilde{\tau}-\hat{\tau}\|_{C^{2+\alpha}(\bar{\Omega})} \\
\text { for all } \tilde{\tau}, \hat{\tau} \in \mathcal{B}_{\delta} \text { and } t \in[-1,+1] .
\end{array}
$$

(5) Due to Theorem 5.2 in [Sauvigny 2012b, Chapter 9], the linear elliptic operator $\mathcal{L}: \mathcal{B} \rightarrow C^{\alpha}(\bar{\Omega})$ satisfies the Schauder estimate

$$
\|\tau\|_{C^{2+\alpha}(\bar{\Omega})} \leq \theta\|\mathcal{L} \tau\|_{C^{\alpha}(\bar{\Omega})} \quad \text { for all } \tau \in \mathcal{B}
$$

where $\theta>0$ represents the $S$ chauder constant. Consequently, $\mathcal{L}$ possesses an inverse $\mathcal{L}^{-1}$ bounded with respect to the respective Hölder norms. With $\delta=\delta(\theta)$ the set $\mathcal{L}\left(\mathcal{B}_{\delta}\right)$ contains a ball of radius $\theta^{-1} \delta$ within the Banach space $C^{\alpha}(\bar{\Omega})$. When we remember (3-24) with $\varepsilon=\varepsilon(\theta)$, we can transform (3-23) into the fixed point equation

$$
\tau=\mathcal{L}^{-1} \circ \mathcal{M}_{t}(\tau), \quad \text { with } \tau \in \mathcal{B}_{\delta} \text { for all }-\varepsilon \leq t \leq+\varepsilon .
$$

The nonlinear operator $\mathcal{L}^{-1} \circ \mathcal{M}_{t}: \mathcal{B}_{\delta} \rightarrow \mathcal{B}_{\delta}$ yields a contraction

$$
\begin{aligned}
& \left\|\mathcal{L}^{-1} \circ \mathcal{M}_{t}(\tilde{\tau})-\mathcal{L}^{-1} \circ \mathcal{M}_{t}(\hat{\tau})\right\|_{C^{2+\alpha}(\bar{\Omega})} \leq \frac{1}{2}\|\tilde{\tau}-\hat{\tau}\|_{C^{2+\alpha}(\bar{\Omega})} \\
& \text { for all } \tilde{\tau}, \hat{\tau} \in \mathcal{B}_{\delta} \text { and }-\varepsilon \leq t \leq+\varepsilon
\end{aligned}
$$

due to (3-25) and (3-27). Banach's fixed point theorem furnishes a unique solution $\tau \in \mathcal{B}_{\delta}$ of the equation (3-28) for all $-\varepsilon \leq t \leq+\varepsilon$.

With the aid of the uniformization method, we shall estimate the area of the solutions for our Dirichlet problem. Let $\Omega \subset \mathbb{R}^{2}$ denote a $C^{2+\alpha}$-Jordan domain with the positive boundary distribution $\phi: \partial \Omega \rightarrow(0,+\infty)$ of the class $C^{2+\alpha}$. We define the Jordan contour

$$
\Gamma(\phi):=\left\{(x \phi(x, y), y \phi(x, y), \phi(x, y)) \in \mathbb{R}^{3} \mid(x, y) \in \partial \Omega\right\}
$$

and the area $M(\phi)>0$ of the conical surface

$$
S(\phi):=\left\{(\lambda x \phi(x, y), \lambda y \phi(x, y), \lambda \phi(x, y)) \in \mathbb{R}^{3} \mid(x, y) \in \partial \Omega, \lambda \in(0,1)\right\} .
$$

With the logarithmic mean curvature (2-12) let us define the mean curvature $H$ due to (2-13) on the cone $\mathcal{C}(\Omega)$. We consider a solution $\varrho=\varrho(x, y) \in \boldsymbol{P}(\Omega, \phi, H)$. 
The associate surface (2-3) possesses the area $A(\varrho)$ and the volume $V(\varrho)$ of the conical domain

$$
G(\varrho):=\left\{(\lambda x \varrho(x, y), \lambda y \varrho(x, y), \lambda \varrho(x, y)) \in \mathbb{R}^{3} \mid(x, y) \in \Omega, \lambda \in(0,1)\right\} .
$$

This $H$-surface has the minimal mean curvature

$$
m(\varrho):=\inf _{(x, y) \in \Omega} H(x \varrho(x, y), y \varrho(x, y), \varrho(x, y)) \in \mathbb{R} .
$$

Lemma 3.2 (area estimate). We can estimate the area $A(\varrho)$ of a solution $\varrho$ for the Dirichlet problem $\boldsymbol{P}(\Omega, \phi, H)$ in

$$
A(\varrho) \leq-3 m(\varrho) V(\varrho)+M(\phi)
$$

by the minimal mean curvature $m(\varrho)$ as well as the volume $V(\varrho)$ of the conical domain $G(\varrho)$ for the solution, and by the area $M(\phi)$ of the given conical surface $S(\phi)$.

Proof. Let us introduce conformal parameters into the surface (2-3). Then we obtain a parametric $H$-surface $X(u, v)=(x(u, v), y(u, v), z(u, v)): \bar{B} \rightarrow \mathbb{R}^{3} \in C^{2+\alpha}(\bar{B})$ which is regular on the closure of the unit disc $B:=\left\{(u, v) \in \mathbb{R}^{2} \mid u^{2}+v^{2}<1\right\}$ in the differential-geometric sense. More precisely, we have the following conditions:

$$
\begin{gathered}
\Delta X(u, v)=2 H(X(u, v)) X_{u} \wedge X_{v}, \quad\left|X_{u}\right|^{2}=\left|X_{v}\right|^{2}>0, \\
X_{u} \cdot X_{v}=0, \quad X \cdot X_{u} \wedge X_{v}>0 \quad \text { on } \bar{B} .
\end{gathered}
$$

With the aid of triple products $(\cdot, \cdot, \cdot)$ we calculate

$$
2 H(X)\left(X, X_{u}, X_{v}\right)=X \cdot \Delta X=\left(X \cdot X_{u}\right)_{u}+\left(X \cdot X_{v}\right)_{v}-|\nabla X|^{2} \quad \text { on } \bar{B}
$$

and obtain

$$
\frac{1}{2}|\nabla X|^{2}=-H(X)\left(X, X_{u}, X_{v}\right)+\frac{1}{2}\left\{\left(X \cdot X_{u}\right)_{u}+\left(X \cdot X_{v}\right)_{v}\right\} \quad \text { on } \bar{B} .
$$

Let us denote the exterior normal to the unit disc by $v: \partial B \rightarrow S^{1}$. Furthermore, we use the arc length $\sigma$ and the line element $d \sigma$ on $\partial B$. Then we integrate (3-33) as follows:

$$
\begin{aligned}
A(\varrho) & =\frac{1}{2} \iint_{B}|\nabla X|^{2} d u d v \\
& =\iint_{B}-H(X)\left(X, X_{u}, X_{v}\right) d u d v+\frac{1}{2} \int_{\partial B}\left(X \cdot X_{v}\right) d \sigma .
\end{aligned}
$$

We use the positive orientation of the conformal parameters as well as the minimal mean curvature (3-30) in order to estimate the two-dimensional integral on the righthand side. Denoting by $N(u, v)$ the unit normal, the conformal parametrization yields the identity

$$
X_{\sigma} \wedge N=X_{v} \quad \text { on } \partial B
$$


which we use in (3-34) for the one-dimensional integral. Thus we obtain

$$
\begin{aligned}
A(\varrho) & \leq-m(\varrho) \iint_{B}\left(X, X_{u}, X_{v}\right) d u d v+\frac{1}{2} \int_{\partial B}\left(X, X_{\sigma}, N\right) d \sigma \\
& \leq-m(\varrho) \iint_{B}\left(X, X_{u}, X_{v}\right) d u d v+\frac{1}{2} \int_{\partial B}\left|X \wedge X_{\sigma}\right| d \sigma \\
& =-m(\varrho) \iint_{B}\left(X, X_{u}, X_{v}\right) d u d v+M(\phi) .
\end{aligned}
$$

Then we apply the Gaussian integral theorem to the conical domain $G(\varrho)$ and the vector field

$$
W(x, y, z):=(x, y, z), \quad(x, y, z) \in G(\varrho),
$$

which is tangential on the conical boundary $\overline{S(\phi)}=\partial G(\varrho) \cap \partial \mathcal{C}(\Omega)$. Therefore, we receive the expression

$$
\iint_{B}\left(X, X_{u}, X_{v}\right) d u d v=\iiint_{G(\varrho)} \operatorname{div} W(x, y, z) d x d y d z=3 V(\varrho) .
$$

We insert (3-36) into (3-35) and obtain with

$$
A(\varrho) \leq-3 m(\varrho) V(\varrho)+M(\phi)
$$

the final estimate.

Remark. Originally, R. Finn [1954] established a priori estimates of the area for graphs of minimal surface type. E. Heinz [1971] proved such an estimate for graphs of prescribed mean curvature. Here we refer our readers to Proposition 1.2 in [Sauvigny 2012b, Chapter 13].

\section{Some results on Radó's minimal surface equation}

In this section, we consider the special case $H \equiv 0 \equiv D$. With the aid of Plateau's problem, we can solve Dirichlet's problem for the PDE (2-17) with vanishing righthand side. This has already been proposed by Radó [1932] (compare [Nitsche 1975, $\S 402])$. However, we shall apply alternative methods from my dissertation [Sauvigny 1982] and book with Dierkes and Hildebrandt [Dierkes et al. 2010a, §§5.1-5.3], in order to realize that the central projection is one-to-one. The $n$-dimensional situation has been studied by E. Tausch [1981] using nonparametric methods.

By variational methods we establish the following theorem.

Theorem 4.1 (solution of $\boldsymbol{P}(\Omega, \phi, 0)$ ). Let $\Omega \subset \mathbb{R}^{2}$ denote a convex $C^{2+\alpha}$-Jordan domain and let $\phi: \partial \Omega \rightarrow(0,+\infty)$ denote a positive $C^{2+\alpha}$-boundary distribution with $0<\alpha<1$. Then the Dirichlet problem $\boldsymbol{P}(\Omega, \phi, 0)$ possesses exactly one solution $\varrho(x, y),(x, y) \in \bar{\Omega}$. 
Proof. (1) We solve Plateau's problem for the regular $C^{2+\alpha}$-Jordan contour

$$
\Gamma:=\left\{(x \phi(x, y), y \phi(x, y), \phi(x, y)) \in \mathbb{R}^{3} \mid(x, y) \in \partial \Omega\right\}
$$

with a parametric minimal surface $X(u, v)=(x(u, v), y(u, v), z(u, v)): \bar{B} \rightarrow \mathbb{R}^{3}$. This surface is defined on the closure of the unit disc $B:=\left\{(u, v) \in \mathbb{R}^{2} \mid u^{2}+v^{2}<1\right\}$ and satisfies the Laplace equation

$$
\Delta X(u, v)=0, \quad(u, v) \in B .
$$

Its isothermal first fundamental form

$$
d s^{2}=X_{u}^{2} d u^{2}+2 X_{u} \cdot X_{v} d u d v+X_{v}^{2} d v^{2}=E(u, v)\left(d u^{2}+d v^{2}\right), \quad(u, v) \in \bar{B},
$$

might only degenerate at isolated branch points of $X$, and its unit normal $N(u, v)$, $(u, v) \in \bar{B}$, exists within the class $C^{2}(B) \cap C^{1}(\bar{B})$ subject to the Schwarzian differential equation

$$
\Delta N(u, v)-2 E(u, v) K(u, v) N(u, v)=0, \quad(u, v) \in B .
$$

Here $K(u, v) \leq 0$ denotes the Gaussian curvature of the metric $d s^{2}$ at regular points $(u, v)$. The differential equation (4-1) can be found in Hilfssatz 1 and Satz 1 of [Sauvigny 1982] (see [Dierkes et al. 2010a, §5.1, Theorem 1] as well). The necessary investigations on the regularity and branch points of $H$-surfaces are contained in Chapter 2 of the treatise [Dierkes et al. 2010b] by Dierkes, Hildebrandt, and A. Tromba.

(2) The minimal surface $X(\bar{B})$ lies in the convex hull of its bounding contour $\Gamma$, where the latter is situated on the boundary of the convex cone $\mathcal{C}(\Omega)$, outside its vertex. This implies the inclusions

$$
X(B) \subset \mathcal{C}(\Omega) \text { and } X(\partial B) \subset \partial \mathcal{C}(\Omega) \backslash\{(0,0,0)\} .
$$

The arguments from [Sauvigny 1982, §2] show that the minimal surface approaches the bounding cone $\partial \mathcal{C}(\Omega)$ transversally and does not possess boundary branch points. When we consider the auxiliary function

$$
\theta(u, v):=N(u, v) \cdot X(u, v), \quad(u, v) \in \bar{B},
$$

we obtain the boundary condition

$$
\theta(u, v)>0, \quad(u, v) \in \partial B .
$$

With the aid of (4-1), we derive the PDE for our auxiliary function

(4-4) $\Delta \theta(u, v)$

$$
\begin{aligned}
& =(\Delta N(u, v)) \cdot X(u, v)+2 \nabla N(u, v) \cdot \nabla X(u, v)+N(u, v) \cdot(\Delta X(u, v)) \\
& =X(u, v) \cdot \Delta N(u, v)-N(u, v) \cdot \Delta X(u, v)=2 E(u, v) K(u, v) \theta(u, v),
\end{aligned}
$$


for $(u, v) \in B$. Before we have fixed three points on the contour $\Gamma$, such that the boundary representation is positive-oriented with respect to the projection plane $\mathcal{E}$.

(3) Now the metric $d s^{2}$ is stable in the following sense:

$$
\begin{aligned}
\iint_{B}|\nabla \psi(u, v)|^{2} d u d v & \\
& \geq-2 \iint_{B} E(u, v) K(u, v) \psi(u, v)^{2} d u d v \text { for all } \psi \in C_{0}^{1}(B) .
\end{aligned}
$$

This stability condition has been established in [Sauvigny 1982, §3] by the areaminimizing property for the solutions of Plateau's problem. Due to Hilfssatz 6 of [Sauvigny 1982] (compare [Dierkes et al. 2010a, §5.3, Proposition 1]), we obtain

$$
\theta(u, v)>0, \quad(u, v) \in \bar{B} .
$$

On the basis of the property (4-6), we can exclude interior branch points for our minimal surface by a winding number argument. Therefore, the surface

$$
X: \bar{B} \rightarrow \mathcal{C}(\bar{\Omega})
$$

represents a minimal embedding, with one-to-one central projection onto the plane $\mathcal{E}$, which bounds the contour $\Gamma$. Thus we have solved the Dirichlet problem $\boldsymbol{P}(\Omega, \phi, 0)$.

Finally, we classify the entire solutions of Radó's partial differential equation.

Theorem 4.2 (Bernstein-type result). Let $\varrho=\varrho(x, y) \in C^{2}\left(\mathbb{R}^{2},(0,+\infty)\right)$ represent a positive solution of the minimal surface equation in central projection

$$
\begin{aligned}
& \left(\varrho_{y}^{2} \cdot\left(x^{2}+y^{2}+1\right)+2 \varrho y \varrho_{y}+\varrho^{2}\right) \varrho_{x x} \\
& \quad-2\left(\varrho_{x} \varrho_{y} \cdot\left(x^{2}+y^{2}+1\right)+\varrho\left[y \varrho_{x}+x \varrho_{y}\right]\right) \varrho_{x y} \\
& \quad+\left(\varrho_{x}^{2} \cdot\left(x^{2}+y^{2}+1\right)+2 \varrho x \varrho_{x}+\varrho^{2}\right) \varrho_{y y}(x, y)-2 \varrho|\nabla \varrho(x, y)|^{2}=0,
\end{aligned}
$$

for $(x, y) \in \mathbb{R}^{2}$. Then it follows that $\varrho(x, y)=c$ for all $(x, y) \in \mathbb{R}^{2}$, with a positive constant $0<c<\infty$.

Proof. (1) We define the complete minimal embedding

$$
X(x, y):=(x \varrho(x, y), y \varrho(x, y), \varrho(x, y)), \quad(x, y) \in \mathbb{R}^{2},
$$

in the Euclidean space $\mathbb{R}^{3}$. For an arbitrary radius $0<R<+\infty$, we consider the geodesic disc about the center $X_{0}:=X(0,0)$ parametrized over the domain $(0,0) \in D_{R} \subset \mathbb{R}^{2}$. Into this minimal disc $X(x, y),(x, y) \in D_{R}$, we introduce conformal parameters and obtain the parametric minimal surface $X(u, v),(u, v) \in B$ with its unit normal $N(u, v),(u, v) \in B$. 
(2) The auxiliary function

$$
\theta(u, v):=N(u, v) \cdot X(u, v), \quad(u, v) \in B,
$$

satisfies the conditions

$$
\begin{aligned}
\Delta \theta(u, v)-2 E(u, v) K(u, v) \theta(u, v) & =0, & & (u, v) \in B, \\
\theta(u, v) & >0, & (u, v) & \in \bar{B} .
\end{aligned}
$$

The arguments of Theorem 1 in [Dierkes et al. 2010a, §5.4] show that our minimal surface $X(u, v),(u, v) \in B$, is stable in the sense of the inequality (4-5). This property holds true for the discs of all radii $0<R<+\infty$ about $X_{0}$.

(3) Theorem 3 in [Dierkes et al. 2010a, §5.5] shows that the surface (4-8) represents a plane within the half-space $z>0$. It follows that $\varrho(x, y)=c$ for all $(x, y) \in \mathbb{R}^{2}$, with a positive constant $0<c<+\infty$.

\section{Large $H$-surfaces bounding extreme contours}

We return to surfaces of prescribed mean curvature with compact support and solve the associate Dirichlet problem on convex domains. We use the deformation method, which is presented in [Sauvigny 2012b, Chapter 13, §1] for the nonparametric $H$ surface equation.

Lemma 5.1 (nondegeneracy result). Suppose $X(u, v):=(x(u, v), y(u, v), z(u, v))$, $(u, v) \in B$, is a parametric $H$-surface, with unit normal $N(u, v),(u, v) \in B$, and suppose its prescribed mean curvature (2-12) in the class $C^{1+\alpha}(\Omega \times \mathbb{R})$ with (2-13) satisfies the monotonicity condition. Furthermore, let the auxiliary function $\theta(u, v):=N(u, v) \cdot X(u, v) \geq 0,(u, v) \in B$, possess a zero $\left(u_{0}, v_{0}\right) \in B$ within this disc. Then the identity $\theta(u, v) \equiv 0,(u, v) \in B$, follows.

Proof. For our prescribed mean curvature $H$, we easily determine the equation

$$
\text { (5-1) } \begin{aligned}
\nabla H(X) \cdot X & =r \cdot \frac{d}{d r}\left\{\frac{D(x, y, \ln r)}{r}\right\}=r \cdot\left\{\frac{D_{z}(x, y, \ln r)}{r^{2}}-\frac{D(x, y, \ln r)}{r^{2}}\right\} \\
& =\frac{D_{z}(x, y, \ln r)}{r}-\frac{D(x, y, \ln r)}{r} \geq-\frac{D(x, y, \ln r)}{r} \\
& =-H(\xi, \eta, \zeta)=-H(X),
\end{aligned}
$$

for all points $X=(\xi, \eta, \zeta)=(r x, r y, r) \in \mathcal{C}(\Omega),(x, y) \in \Omega, 0<r<+\infty$. With the aid of Hilfssatz 1 and Satz 1 in [Sauvigny 1982] (see also [Dierkes et al. 2010a, §5.1, Theorem 1]) and the estimate (5-1), we obtain the following differential inequality 
on the unit disc $B$ for our auxiliary function:

$$
\begin{aligned}
\Delta \theta & (u, v) \\
& =(\Delta N(u, v)) \cdot X(u, v)+2 \nabla N(u, v) \cdot \nabla X(u, v)+N(u, v) \cdot(\Delta X(u, v)) \\
& =X(u, v) \cdot \Delta N(u, v)-N(u, v) \cdot \Delta X(u, v) \\
& =X(u, v) \cdot \Delta N(u, v)-\left.2 E(u, v) H\right|_{X(u, v)} \\
& =-q(u, v) \theta(u, v)-\left.2 E(u, v) \nabla H\right|_{X(u, v)} \cdot X(u, v)-\left.2 E(u, v) H\right|_{X(u, v)} \\
& \leq-q(u, v) \theta(u, v) .
\end{aligned}
$$

Here we have used the potential

$(5-3) q(u, v):=2\left(\left.2 E(u, v) H\right|_{X(u, v)} ^{2}-E K(u, v)-\left.E(u, v) \nabla H\right|_{X(u, v)} \cdot N(u, v)\right)$,

for $(u, v) \in B$. Since a point $\left(u_{0}, v_{0}\right) \in B$ with $\theta\left(u_{0}, v_{0}\right)=0$ exists, the nonnegative function $\theta$ solving (5-2) has to vanish in $B$ identically, due to Hilfssatz 5 in [Sauvigny 1982].

By a nonlinear continuity method we prove the following theorem.

Theorem 5.1 (solution of $\boldsymbol{P}\left(\Omega, \phi, H^{*}\right)$ ). On the convex $C^{2+\alpha}$-Jordan domain $\Omega$ we prescribe the logarithmic mean curvature

$$
D(x, y)=D^{*}(x, y): \Omega \rightarrow \mathbb{R} \in C_{0}^{1+\alpha}(\Omega)
$$

with compact support, subject to the inequalities

$$
\widehat{D}(x, y) \leq D(x, y) \leq 0 \quad \text { for all points }(x, y) \in \Omega .
$$

Denote by $H^{*}$ its homogeneous continuation onto the cone $\mathcal{C}(\Omega)$ due to (2-13). Then the Dirichlet problem $\boldsymbol{P}\left(\Omega, \phi, H^{*}\right)$ of the $H^{*}$-surface equation in central projection possesses a solution $\varrho=\varrho(x, y) \in C^{2+\alpha}(\bar{\Omega})$ for all $C^{2+\alpha}$ functions $\phi: \partial \Omega \rightarrow(0,+\infty)$

Proof. (1) We introduce the positive quantity

$$
r^{*}:=\left(1+b^{2}\right) \cdot \max _{(\xi, \eta) \in \partial \Omega} \phi(\xi, \eta) .
$$

Then we choose a weakly monotonically decreasing function $\chi=\chi(z) \in C^{1}(\mathbb{R},[0,1])$ with the properties

$$
\chi(z)=1 \text { for all } z \in\left(-\infty, \ln r^{*}\right], \quad \chi(z)=0 \text { for all } z \in\left[\ln \left(r^{*}+1\right),+\infty\right) .
$$

Thus we prescribe the logarithmic mean curvature

$$
D=D(x, y, z):=D^{*}(x, y) \cdot \chi(z), \quad(x, y, z) \in \Omega \times \mathbb{R},
$$


on the cylinder $\Omega \times \mathbb{R}$. Due to (2-13), we observe the associate mean curvature $H$ on the cone $\mathcal{C}(\Omega)$ as follows:

$$
\begin{gathered}
H(\xi, \eta, \zeta)=\frac{D^{*}(x, y)}{r}=H^{*}(\xi, \eta, \zeta), \quad(\xi, \eta, \zeta)=(r x, r y, r), \\
\text { with }(x, y) \in \Omega, 0<r \leq r^{*},
\end{gathered}
$$

and

$$
\begin{gathered}
H(\xi, \eta, \zeta)=0, \quad(\xi, \eta, \zeta)=(r x, r y, r), \\
\text { with }(x, y) \in \Omega, r^{*}+1 \leq r<+\infty .
\end{gathered}
$$

(2) For the parameters $1 \leq \lambda<+\infty$ let us consider the Dirichlet problems $\boldsymbol{P}(\Omega, \lambda \phi, H)$ with the mean curvature $H$ being prescribed. These $H$-surfaces with one-to-one central projection bound the contours

$$
\Gamma_{\lambda}:=\left\{(x \lambda \phi(x, y), y \lambda \phi(x, y), \lambda \phi(x, y)) \in \mathbb{R}^{3} \mid(x, y) \in \partial \Omega\right\}
$$

situated on the boundary of our cone. On account of (5-9) we can choose a parameter $1<\lambda^{*}<+\infty$ large enough that the mean curvature $H$ vanishes within the convex hull of the contour $\Gamma_{\lambda^{*}}$. Therefore, we can solve the Dirichlet problem $\boldsymbol{P}\left(\Omega, \lambda^{*} \phi, H\right)$ with the aid of Theorem 4.1.

(3) Now the set

$$
\Lambda:=\left\{\lambda \in\left[1, \lambda^{*}\right] \mid \boldsymbol{P}(\Omega, \lambda \phi, H) \text { possesses a solution }\right\}
$$

is open, since the solutions are stable with respect to small homothetic perturbations of the bounding contour due to Lemma 3.1. Here we use that the logarithmic mean curvature from (5-6) and (5-7) satisfies the monotonicity condition in Definition 3.2. Moreover, the set $\Lambda$ is closed since the property of one-to-one central projection remains valid in the limit. This follows from Lemma 5.1 which requires the monotonicity condition again. Since the cone is convex and the mean curvature vanishes near the boundary, the surfaces approach the conical boundary $\partial \mathcal{C}(\Omega)$ transversally.

(4) In order to establish the compactness of our solutions, we need a joint bound on the area of the surfaces. Here we use the area estimate from Lemma 3.2 as follows: on account of Theorem 3.2 we obtain a bound from below for the mean curvature of our surfaces. Furthermore, this Theorem 3.2 yields a uniform estimate for the volumes appearing in Lemma 3.2.

With the aid of the Courant-Lebesgue lemma and the geometric maximum principle in a local version, we can easily derive a modulus of continuity for our parametric solutions in order to establish the equicontinuity of our functions on the closed 
disc $\bar{B}$. In this context, we use at each point $\left(\xi_{0}, \eta_{0}, \zeta_{0}\right)=\left(r_{0} x_{0}, r_{0} y_{0}, r_{0}\right) \in \mathcal{C}(\bar{\Omega})$, with $\left(x_{0}, y_{0}\right) \in \bar{\Omega}$ and $r_{0}>0$, the conical $\varepsilon$-neighborhood

$$
\begin{aligned}
U_{\varepsilon}\left(\xi_{0},\right. & \left.\eta_{0}, \zeta_{0}\right) \\
:= & \left\{(r x, r y, r) \in \mathbb{R}^{3} \mid(x, y) \in \bar{\Omega} \text { with }\left(x-x_{0}\right)^{2}+\left(y-y_{0}\right)^{2} \leq \varepsilon^{2}\right. \text { and } \\
& \left.\quad r \in \mathbb{R} \text { with }(1-\varepsilon) r_{0} \leq r \leq(1+\varepsilon) r_{0} \mu\left(x_{0}, y_{0}, \varepsilon\right) /\left(1+x^{2}+y^{2}\right)\right\}
\end{aligned}
$$

where we need the function

$$
\mu\left(x_{0}, y_{0}, \varepsilon\right):=\sup \left\{1+x^{2}+y^{2} \mid(x, y) \in \Omega \text { with }\left(x-x_{0}\right)^{2}+\left(y-y_{0}\right)^{2}<\varepsilon^{2}\right\} .
$$

The parametric representation $X(u, v),(u, v) \in \bar{B}$, of a solution $\varrho=\varrho(x, y)$, $(x, y) \in \bar{\Omega}$, for our Dirichlet problem under condition (5-4) is subject to the inclusion principle

$X(\partial \Theta) \subset U_{\varepsilon}\left(\xi_{0}, \eta_{0}, \zeta_{0}\right) \quad \Rightarrow \quad X(\bar{\Theta}) \subset U_{\varepsilon}\left(\xi_{0}, \eta_{0}, \zeta_{0}\right) \quad$ for all domains $\Theta \subset \Omega$,

where we use the proof of Theorem 3.2. Then we can adapt the proof of Theorem 2(iii) in [Dierkes et al. 2010a, \$7.1] and especially Lemma 4 to obtain the desired equicontinuity. Alternatively, we can modify the proofs of Satz 5 and Hilfssatz 10 in [Sauvigny 1982] by using the inclusion principle above.

(5) Now we combine from [Sauvigny 2012b, Chapter 12] the gradient estimate Theorem 2.6 by Heinz and the inner $C^{1+\alpha}$-estimate Theorem 2.7 for the $H$-surface system (3-31), which both require a smallness condition, with the modulus of continuity as in proof of Theorem 5.4(2). Thus we obtain an inner $C^{1+\alpha}$-estimate for our solutions, which implies an interior $C^{2+\alpha}$-estimate via Theorem 4.4 in [Sauvigny 2012b, Chapter 9].

Therefore, we can extract a uniformly convergent subsequence on $\bar{B}$ which converges in $C_{\text {loc }}^{2+\alpha}(B) \cap C^{0}(\bar{B})$ to a solution of Plateau's problem. We invoke the boundary regularity result proved by Heinz [1970] and Hildebrandt (see [Dierkes et al. 2010b, §2.3, Theorem 2]). Thus our limit surface belongs to the Banach space $C^{2+\alpha}(\bar{B})$.

Alternatively, we could control the convergence within the Banach space $C^{2+\alpha}(\bar{B})$ with the aid of [Dierkes et al. 2010b, §2.1, Proposition 2 and Lemma 7; §2.2, Theorem 2], together with Theorems 4.6 and 5.2 from [Sauvigny 2012b, Chapter 9].

(6) Since our set $\Lambda$ is nonempty, we obtain the identity $\Lambda=\left[1, \lambda^{*}\right]$. Therefore, the problem $\boldsymbol{P}(\Omega, \phi, H)$ possesses a solution. Finally, we use Theorem 3.2 and remember (5-5). Consequently, the solution of $\boldsymbol{P}(\Omega, \phi, H)$ lies within the conical section described in (5-8), where the curvatures $H$ and $H^{*}$ coincide. Thus we have found a solution of the Dirichlet problem $\boldsymbol{P}\left(\Omega, \phi, H^{*}\right)$. 
Definition 5.1. To each Jordan domain $\Omega \subset \mathbb{R}^{2}$ and positive boundary distribution $\phi: \partial \Omega \rightarrow(0,+\infty) \in C^{0}(\partial \Omega, \mathbb{R})$ we associate the Jordan contour

$$
\Gamma=\Gamma(\Omega, \phi):=\left\{(x \phi(x, y), y \phi(x, y), \phi(x, y)) \in \mathbb{R}^{3} \mid(x, y) \in \partial \Omega\right\}
$$

with one-to-one central projection onto the curve $\partial \Omega$. We name $\Gamma$ an extreme contour if each point $X_{0}=\left(\xi_{0}, \eta_{0}, \zeta_{0}\right) \in \Gamma$ admits a real number $a=a\left(X_{0}\right)>0$ such that

$$
\Gamma \subset \mathcal{K}(a) \quad \text { and } \quad X_{0} \in \Gamma \cap \partial \mathcal{K}(a)
$$

Here we use the balls of support

$$
\mathcal{K}(a):=\left\{(\xi, \eta, \zeta) \in \mathbb{R}^{3} \mid \xi^{2}+\eta^{2}+(\zeta-a / 2)^{2} \leq a^{2} / 4\right\}
$$

of radius $a / 2>0$ about the center $(0,0, a / 2)$.

Theorem 5.2 (large embedded solutions for Plateau's problem). We have a function $\phi=\phi(x, y): \partial \Omega \rightarrow(0,+\infty) \in C^{2+\alpha}$, on the boundary of a convex $C^{2+\alpha}-$ Jordan domain $\Omega \subset \mathbb{R}^{2}$, such that $\Gamma=\Gamma(\Omega, \phi)$ represents an extreme contour. Furthermore, let us prescribe with $D=D(x, y): \bar{\Omega} \rightarrow \mathbb{R} \in C^{1+\alpha}(\bar{\Omega})$ the logarithmic mean curvature subject to the restriction (5-4) and denote by $H$ its homogeneous continuation onto the cone $\mathcal{C}(\Omega)$ due to (2-13). Then we can solve the Dirichlet problem $\boldsymbol{P}(\Omega, \phi, H)$ by a function $\varrho=\varrho(x, y) \in C^{2+\alpha}(\bar{\Omega})$.

Proof. (1) Approximate $D=D(x, y)$ by functions $D^{k}=D^{k}(x, y): \Omega \rightarrow \mathbb{R} \in C_{0}^{1+\alpha}(\Omega)$ for $k=1,2, \ldots$ within $C_{\text {loc }}^{\alpha}(\Omega)$ which are dominated as follows:

$$
D(x, y) \leq D^{k}(x, y) \leq 0, \quad(x, y) \in \Omega, \quad \text { for all } k \in \mathbb{N} .
$$

Therefore, these functions $D^{k}$ are subject to the restrictions (5-4), and the convergence in $\Omega$ is compactly uniform. Then we denote by $H^{k}$ their continuation onto the cone $\mathcal{C}(\Omega)$ due to (2-13). With the aid of Theorem 5.1 we solve the Dirichlet problems $\boldsymbol{P}\left(\Omega, \phi, H^{k}\right)$ by the functions $\varrho^{k}=\varrho^{k}(x, y) \in C^{2+\alpha}(\bar{\Omega})$ for all $k \in \mathbb{N}$.

(2) We choose the solution $\tilde{\varrho}(x, y),(x, y) \in \bar{\Omega}$, of the problem $\boldsymbol{P}(\Omega, \phi, 0)$ in Theorem 4.1 as a lower barrier function. Now we observe that $\Gamma$ represents an extreme contour. For each $\left(x_{0}, y_{0}\right) \in \partial \Omega$ with the associate point

$$
X_{0}:=\left(x_{0} \phi\left(x_{0}, y_{0}\right), y_{0} \phi\left(x_{0}, y_{0}\right), \phi\left(x_{0}, y_{0}\right)\right) \in \Gamma
$$

we can find a real number $a=a\left(x_{0}, y_{0}\right)>0$ such that the ball $\mathcal{K}(a)$ from (5-15) satisfies the conditions (5-14). Together with the solution

$$
\hat{\varrho}(x, y):=a\left(1+x^{2}+y^{2}\right)^{-1}, \quad(x, y) \in \bar{\Omega},
$$


from Theorem 2.1 as an upper barrier function, we obtain the estimates

$$
\tilde{\varrho}(x, y) \leq \varrho^{k}(x, y) \leq \hat{\varrho}(x, y) \quad \text { for all }(x, y) \in \bar{\Omega} \text { and all } k \in \mathbb{N}
$$

via the method of proof in Theorem 3.2.

(3) The parametric representations of these solutions yield $H^{k}$-surfaces $X^{k}(u, v)$ of equibounded mean curvature and belonging to $C^{2+\alpha}(\bar{B})$. Now we use the arguments in parts (4) and (5) of the proof for Theorem 5.1 and establish the equicontinuity of $\left\{X^{k}\right\}_{k=1,2,3, \ldots}$ on the closed disc by the inclusion principle. With the aid of [Dierkes et al. 2010b, §2.2, Theorem 2 and $\$ 2.3$, Theorem 2] we see that these functions converge to a function $X \in C^{1+\alpha}(\bar{B}, \mathcal{T})$ in isothermal parameters which is situated in the spherical solid

$$
\mathcal{T}:=\left\{Y=(r x, r y, r) \in \mathbb{R}^{3} \mid(x, y) \in \bar{\Omega}, \tilde{\varrho}(x, y) \leq r \leq \hat{\varrho}(x, y)\right\} .
$$

At the point $X_{0} \in \partial \mathcal{T}$ the surfaces associated with the lower and upper barrier functions form an angle $\omega=\omega\left(x_{0}, y_{0}\right) \in(0, \pi)$.

The inclusion $X(\bar{B}) \subset \mathcal{T}$ and the representation $X\left(u_{0}, v_{0}\right)=X_{0} \in \partial \mathcal{T}$ with $\left(u_{0}, v_{0}\right) \in \partial B$ imply that the point $\left(u_{0}, v_{0}\right)$ does not constitute a branch point of $X$. Otherwise the local expansion there would imply that the surface $X$ protrudes from $\mathcal{T}$ - an evident contradiction.

Furthermore, the inclusion $X(B) \subset \mathcal{C}(\Omega)$ holds true. If $X\left(u_{0}, v_{0}\right)=X_{0} \in \partial \mathcal{T}$ were true for a point $\left(u_{0}, v_{0}\right) \in B$, the local expansion of $X$ would force the surface to protrude from $\mathcal{T}$ - which is impossible. Since the boundary point $X_{0}$ can be chosen arbitrarily on $\Gamma$, the inclusion above and the exclusion of branch points on $\partial B$ is established.

For the local expansions, we refer our readers to Theorem 2 and Corollary 2 in [Dierkes et al. 2010b, §3.1] and to the original paper by Heinz [1970].

(4) The functions $X^{k}(u, v) \in C^{2+\alpha}(\bar{B})$ satisfy the nonlinear elliptic systems

$$
\Delta X^{k}(u, v)=2 H^{k}\left(X^{k}(u, v)\right) X_{u}^{k} \wedge X_{v}^{k} \quad \text { on } \bar{B} \text { for } k=1,2, \ldots
$$

Since the mean curvatures $H^{k}=H^{k}(\xi, \eta, \zeta): \mathcal{C}(\Omega) \rightarrow \mathbb{R}$ converge compactly uniformly in the open cone $\mathcal{C}(\Omega)$ and the surfaces $X^{k}=X^{k}(u, v): \bar{B} \rightarrow \overline{\mathcal{C}(\Omega)}$ converge due to (3) uniformly on $\bar{B}$ to the continuous function $X: \bar{B} \rightarrow \overline{\mathcal{C}(\Omega)}$ with the property $X(B) \subset \mathcal{C}(\Omega)$, we see the limit relation

$$
\lim _{k \rightarrow \infty} H^{k}\left(X^{k}(u, v)\right)=H(X(u, v)) \quad \text { for all }(u, v) \in B .
$$

Since the relation (5-20) occurs within $C_{\mathrm{loc}}^{\alpha}(B)$ and a modulus of continuity in (3) has been established, we can use the arguments from part (5) in the proof of Theorem 5.1. Consequently, the functions $X^{k}$ converge within the space $C_{\mathrm{loc}}^{2+\alpha}(B)$ 
to the $H$-surface

$$
X=X(u, v) \in C^{2+\alpha}(B) \cap C^{0}(\bar{B})
$$

bounding the regular $C^{2+\alpha}$-contour $\Gamma$.

(5) We invoke Theorem 2 in [Dierkes et al. 2010b, §2.3] again and see that $X=X(u, v) \in C^{2+\alpha}(\bar{B})$. Furthermore, Lemma 5.1 guarantees that our limit surface satisfies

$$
X(u, v) \cdot N(u, v)>0, \quad(u, v) \in \bar{B},
$$

where we use the inclusion $X(\bar{B}) \subset \mathcal{T}$ at the boundary. By a winding number argument, we can easily exclude the interior branch points, and the $H$-surface $X: \bar{B} \rightarrow \mathbb{R}^{3}$ is a differential-geometrically regular surface. Finally, the nonparametric representation of this surface $\varrho=\varrho(x, y) \in C^{2+\alpha}(\bar{\Omega})$ solves the Dirichlet problem $\boldsymbol{P}(\Omega, \phi, H)$.

Remark. For arbitrary $a>0$, let us consider a regular $C^{2+\alpha}$-Jordan contour on the boundary of the ball $\mathcal{K}(a)$ with a one-to-one and convex central projection onto the plane $\mathcal{E}$. Due to Theorem 5.2 above, we can construct for all nonpositive curvatures $H$ greater or equal to the mean curvature of the upper hemisphere an $H$-surface bounding the contour $\Gamma$ with one-to-one projection onto $\mathcal{E}$. Since these surfaces include the large spherical caps, we receive large embedded solutions of Plateau's problem. We have to distinguish our considerations from the investigations of H. Brézis and M. Coron [1984] or independently of M. Struwe [1985]. For constant $H$ they construct two solutions of Rellich's $H$-surface system by variational methods and obtain two not necessarily immersed $H$-surfaces which solve Plateau's problem for the same contour.

\section{The Dirichlet problem $P\left(\Omega_{b}, \phi, H\right)$ on discs}

In this section we concentrate on circular cones $\mathcal{C}\left(\Omega_{b}\right)$ associated with the discs

$$
\Omega_{b}:=\left\{(x, y) \in \mathbb{R}^{2} \mid x^{2}+y^{2}<b^{2}\right\}
$$

of radius $0<b<+\infty$ about the origin. One can easily prove the following lemma. Lemma 6.1 (boundary curvature). For arbitrary radii $0<b<+\infty$ we parametrize the circular cones $\partial \mathcal{C}\left(\Omega_{b}\right)$ by

$$
Y=Y(r, t):=(r b \cos t, r b \sin t, r), \quad 0<r<+\infty, \quad 0 \leq t<2 \pi .
$$

Their mean curvature with respect to the interior normal is given by

$$
H_{b}(r, t)=\frac{1}{2 r b \sqrt{1+b^{2}}}, \quad 0<r<+\infty, \quad 0 \leq t<2 \pi .
$$


Theorem 6.1 (solution of $\left.\boldsymbol{P}\left(\Omega_{b}, \phi, H\right)\right)$. On the closed disc $\bar{\Omega}_{b}$ of radius $0<b<\infty$, let us prescribe the logarithmic mean curvature $D(x, y) \in C^{1+\alpha}\left(\bar{\Omega}_{b}\right)$ subject to the inequalities (5-4) and the estimate

$$
-\frac{1}{2 b \sqrt{1+b^{2}}}<D(x, y) \leq 0 \text { for all points }(x, y) \in \partial \Omega_{b} .
$$

We denote by $H$ its homogeneous continuation onto the circular cone $\mathcal{C}\left(\Omega_{b}\right)$ due to (2-13). Then the Dirichlet problem $\boldsymbol{P}\left(\Omega_{b}, \phi, H\right)$ possesses a solution

$$
\varrho=\varrho(x, y) \in C^{2+\alpha}\left(\Omega_{b}\right) \cap C^{0}\left(\bar{\Omega}_{b}\right)
$$

for all continuous boundary distributions $\phi: \partial \Omega_{b} \rightarrow(0,+\infty)$.

Proof. (1) As in the proof of Theorem 5.2, let us approximate $D=D(x, y)$ by the functions

$$
D^{k}=D^{k}(x, y): \Omega_{b} \rightarrow \mathbb{R} \in C_{0}^{1+\alpha}\left(\Omega_{b}\right) \quad \text { for } k=1,2, \ldots
$$

within $C_{\text {loc }}^{\alpha}\left(\Omega_{b}\right)$, dominated due to (5-16) when we replace the domain $\Omega$ with $\Omega_{b}$. We denote by $H^{k}$ their continuation onto the circular cone $\mathcal{C}\left(\Omega_{b}\right)$ due to (2-13) and approximate the continuous boundary distribution $\phi: \partial \Omega_{b} \rightarrow(0,+\infty) \in C^{0}$ uniformly by the sequence $\phi^{k}: \partial \Omega_{b} \rightarrow(0,+\infty) \in C^{2+\alpha}$ for $k=1,2, \ldots$

With the aid of Theorem 5.1, we solve the Dirichlet problems $\boldsymbol{P}\left(\Omega_{b}, \phi^{k}, H^{k}\right)$ by the functions $\varrho^{k}=\varrho^{k}(x, y) \in C^{2+\alpha}\left(\bar{\Omega}_{b}\right)$ for all $k \in \mathbb{N}$. In the parametric form we receive $H^{k}$-surfaces $X^{k}(u, v) \in C^{2+\alpha}(\bar{B})$ bounding the Jordan contours $\Gamma^{k}:=\Gamma\left(\Omega_{b}, \phi_{k}\right)$ from (5-13). By an area estimate as in part (4) of the proof of Theorem 5.1, we select a subsequence of $\left\{X^{k}(u, v)\right\}_{k=1,2, \ldots}$ which is uniformly convergent on $\bar{B}$ to the limit

$$
X=X(u, v) \in C^{0}\left(\bar{B}, \mathcal{C}\left(\bar{\Omega}_{b}\right)\right) .
$$

(2) Let us take a point $(x, y)=(b \cos t, b \sin t) \in \partial \Omega_{b}$ with an appropriate $0 \leq t<2 \pi$ and a number $r>0$, such that we obtain the boundary point $(r x, r y, r) \in \partial \mathcal{C}\left(\Omega_{b}\right)$ of the cone. We use the balls

$$
K_{\delta}(r x, r y, r):=\left\{(\xi, \eta, \zeta) \in \mathbb{R}^{3} \mid(\xi-r x)^{2}+(\eta-r y)^{2}+(\zeta-r)^{2}<\delta^{2}\right\}
$$

about this point of radius $\delta=\delta(r x, r y, r)>0$, which we shall choose sufficiently small. Now we need circular cylinders of curvature $h>0$ which are generated as images of the standard cylinder

$$
\mathcal{S}_{h}:=\left\{(\xi, \eta, \zeta) \in \mathbb{R}^{3} \mid \xi^{2}+\eta^{2}<1 / 4 h^{2}\right\}
$$

of curvature $h$ under an appropriate rotation and translation. 
At the point $(r x, r y, r)$ we use as the cylinder of support $\mathcal{S}_{h}(r x, r y, r)$ the circular cylinder of curvature

$$
h:=\frac{1}{2 r}\left(\frac{1}{2 b \sqrt{1+b^{2}}}-D(x, y)\right) \in\left(-\frac{1}{r} D(x, y), H_{b}(r, t)\right)
$$

with the properties

$$
\begin{gathered}
\mathcal{C}\left(\Omega_{b}\right) \cap K_{\delta}(r x, r y, r) \subset \mathcal{S}_{h}(r x, r y, r) \cap K_{\delta}(r x, r y, r), \\
\partial \mathcal{C}\left(\Omega_{b}\right) \cap \partial \mathcal{S}_{h}(r x, r y, r) \cap K_{\delta}(r x, r y, r)=L_{(x, y)} \cap K_{\delta}(r x, r y, r) .
\end{gathered}
$$

Here we have to apply our Lemma 6.1 from above. For the prescribed mean curvature, the estimate

$$
|H(\xi, \eta, \zeta)| \leq h \quad \text { at all points }(\xi, \eta, \zeta) \in \mathcal{C}\left(\bar{\Omega}_{b}\right) \cap K_{\delta}(r x, r y, r)
$$

holds true.

(3) The limit surface $X(u, v)$ from (1) cannot touch the cone $\partial \mathcal{C}\left(\Omega_{b}\right)$ at an interior point. If this happened, we could find a point $\left(u_{0}, v_{0}\right) \in B$ such that $X\left(u_{0}, v_{0}\right)=(r x, r y, r)$ holds true for a boundary point $(r x, r y, r) \in \partial \mathcal{C}\left(\Omega_{b}\right)$ considered in (2) above. Now we use Hildebrandt's geometric maximum principle for $H$-surfaces in circular cylinders, presented in Hilfssatz 3 in [Sauvigny 1982] or Proposition 1.6 in [Sauvigny 2012b, Chapter 13].

Transforming the setting into the standard cylinder (6-4) by rotation and translation, we show in (4) that the continuous auxiliary function

$$
\begin{gathered}
\Psi(u, v):=x(u, v)^{2}+y(u, v)^{2}, \quad(u, v) \in B, \\
\text { with }\left(u-u_{0}\right)^{2}+\left(v-v_{0}\right)^{2}<\epsilon^{2},
\end{gathered}
$$

is subharmonic in the sense of mean values, where $\epsilon>0$ is sufficiently small. Due to Theorem 2.9 in [Sauvigny 2012a, Chapter 5], the function $\Psi$ is subject to the maximum principle. Therefore, the surface $X$ would locally coincide with the bounding cylinder and protrude from $\mathcal{C}\left(\bar{\Omega}_{b}\right)$, which is impossible. Consequently, we have

$$
X(B) \subset \mathcal{C}\left(\Omega_{b}\right) .
$$

(4) Now we prove that the function $\Psi$ is subharmonic: for $k=1,2, \ldots$ we consider the approximate auxiliary functions

$$
\begin{gathered}
\Psi^{k}(u, v):=x^{k}(u, v)^{2}+y^{k}(u, v)^{2}, \quad(u, v) \in B, \\
\text { with }\left(u-u_{0}\right)^{2}+\left(v-v_{0}\right)^{2}<\epsilon^{2},
\end{gathered}
$$

associated with the solutions $X^{k} \in C^{2+\alpha}\left(\bar{B}, \mathcal{C}\left(\bar{\Omega}_{b}\right)\right)$ of the $H$-surface system (5-19). Since their mean curvatures $H^{k}$ are equally bounded as in (6-7) due to (5-16), these 
functions satisfy

$$
\Delta \Psi^{k}(u, v) \geq 0, \quad(u, v) \in B, \quad \text { with }\left(u-u_{0}\right)^{2}+\left(v-v_{0}\right)^{2}<\epsilon^{2},
$$

by Hildebrandt's maximum principle cited above. The functions $X^{k}$ and $\Psi^{k}$ converge uniformly, and consequently the subharmonic property (6-11) for $\Psi^{k}$ - in the mean-value sense - is transferred to the limit function $\Psi$.

(5) As we have seen in part (4) of the proof of Theorem 5.2, the inclusion (6-9) implies that the convergence of our sequence $X^{k}(u, v) \in C^{2+\alpha}(\bar{B}), k=1,2, \ldots$, occurs in the space $C_{\mathrm{loc}}^{2+\alpha}(B) \cap C^{0}(\bar{B})$ to the limit surface

$$
X=X(u, v) \in C^{2+\alpha}(B) \cap C^{0}(\bar{B}) .
$$

Lemma 5.1 guarantees that our surface satisfies

$$
X(u, v) \cdot N(u, v)>0, \quad(u, v) \in B .
$$

Therefore, the surface $X$ has a one-to-one central projection onto the plane $\mathcal{E}$ and possesses the nonparametric representation

$$
\varrho=\varrho(x, y) \in C^{2+\alpha}\left(\Omega_{b}\right) \cap C^{0}\left(\bar{\Omega}_{b}\right)
$$

solving the Dirichlet problem $\boldsymbol{P}\left(\Omega_{b}, \phi, H\right)$.

\section{Acknowledgements}

I cordially thank Professor Dr. Dr.h.c. Robert Finn (Stanford University) for his interest in my investigations. I am also very grateful to Privatdozent Dr. Ruben Jakob (Eberhard Karls Universität Tübingen) for many valuable suggestions. His detailed comments rendered my paper more readable and the proofs of Lemma 3.1 and Theorems 5.1 and 5.2 more rigorous. Finally I would like to thank the production editor Silvio Levy and his staff for their excellent work.

\section{References}

[Brézis and Coron 1984] H. Brézis and J.-M. Coron, "Multiple solutions of $H$-systems and Rellich's conjecture", Comm. Pure Appl. Math. 37:2 (1984), 149-187. MR 85i:53010 Zbl 0537.49022

[Dierkes et al. 2010a] U. Dierkes, S. Hildebrandt, and F. Sauvigny, Minimal surfaces, 2nd ed., Grundlehren der Mathematischen Wissenschaften 339, Springer, Heidelberg, 2010. MR 2012b:49002 Zbl 1213.53002

[Dierkes et al. 2010b] U. Dierkes, S. Hildebrandt, and A. J. Tromba, Regularity of minimal surfaces, 2nd ed., Grundlehren der Mathematischen Wissenschaften 340, Springer, Heidelberg, 2010. MR 2012b:49003 Zbl 1213.53003

[Finn 1954] R. Finn, "On equations of minimal surface type", Ann. of Math. (2) 60 (1954), 397-416. MR 16,592b Zbl 0058.32501 
[Heinz 1970] E. Heinz, "Über das Randverhalten quasilinearer elliptischer Systeme mit isothermen Parametern”, Math. Z. 113 (1970), 99-105. MR 41 \#7288 Zbl 0176.41004

[Heinz 1971] E. Heinz, "Interior gradient estimates for surface $z=f(x, y)$ with prescribed mean curvature", J. Differential Geometry 5:1-2 (1971), 149-157. MR 44 \#7125 Zbl 0212.44001

[Nitsche 1975] J. C. C. Nitsche, Vorlesungen über Minimalfä̈chen, Grundlehren der mathematischen Wissenschaften 199, Springer, Berlin, 1975. MR 56 \#6533 Zbl 0319.53003

[Radó 1932] T. Radó, "Contributions to the theory of minimal surfaces", Acta Sci. Math. (Szeged) 6:1 (1932), 1-20. Zbl 0005.17901

[Sauvigny 1982] F. Sauvigny, "Flächen vorgeschriebener mittlerer Krümmung mit eineindeutiger Projektion auf eine Ebene”, Math. Z. 180:1 (1982), 41-67. MR 83j:53004 Zbl 0465.53003

[Sauvigny 2012a] F. Sauvigny, Partial differential equations, 1: Foundations and integral representations, 2nd ed., Springer, Berlin, 2012. MR 2007c:35001 Zbl 1246.35001

[Sauvigny 2012b] F. Sauvigny, Partial differential equations, 2: Functional analytic methods, 2nd ed., Springer, London, 2012. MR 2012m:35001 Zbl 1246.35002

[Serrin 1969] J. Serrin, "On surfaces of constant mean curvature which span a given space curve", Math. Z. 112 (1969), 77-88. MR 40 \#3447 Zbl 0182.24001

[Struwe 1985] M. Struwe, "Large $H$-surfaces via the mountain-pass-lemma", Math. Ann. 270:3 (1985), 441-459. MR 86d:58024 Zbl 0582.58010

[Tausch 1981] E. Tausch, "The $n$-dimensional least area problem for boundaries on a convex cone", Arch. Rational Mech. Anal. 75:4 (1981), 407-416. MR 82e:53014 Zbl 0477.53006

Received August 6, 2015. Revised August 11, 2015.

\section{FRIEDRICH SAUVIGNY}

MATHEMATISCHES INSTITUT

Brandenburgische TeChnische Universität Cottbus - SEnFtenberg

Platz DER DEUTSCHEN EINHEIT 1

D-03046 CotTBus

GERMANY

sauvigny@b-tu.de 


\title{
PACIFIC JOURNAL OF MATHEMATICS
}

\author{
msp.org/pjm
}

Founded in 1951 by E. F. Beckenbach (1906-1982) and F. Wolf (1904-1989)

\section{EDITORS}

Don Blasius (Managing Editor)

Department of Mathematics

University of California

Los Angeles, CA 90095-1555

blasius@math.ucla.edu

\author{
Paul Balmer \\ Department of Mathematics \\ University of California \\ Los Angeles, CA 90095-1555 \\ balmer@math.ucla.edu \\ Robert Finn \\ Department of Mathematics \\ Stanford University \\ Stanford, CA 94305-2125 \\ finn@math.stanford.edu \\ Sorin Popa \\ Department of Mathematics \\ University of California \\ Los Angeles, CA 90095-1555 \\ popa@math.ucla.edu
}

\author{
Vyjayanthi Chari \\ Department of Mathematics \\ University of California \\ Riverside, CA 92521-0135 \\ chari@math.ucr.edu \\ Kefeng Liu \\ Department of Mathematics \\ University of California \\ Los Angeles, CA 90095-1555 \\ liu@math.ucla.edu \\ Jie Qing \\ Department of Mathematics \\ University of California \\ Santa Cruz, CA 95064 \\ qing@ cats.ucsc.edu
}

\section{PRODUCTION}

Silvio Levy, Scientific Editor, production@msp.org

\section{SUPPORTING INSTITUTIONS}

ACADEMIA SINICA, TAIPEI

CALIFORNIA INST. OF TECHNOLOGY

INST. DE MATEMÁTICA PURA E APLICADA

KEIO UNIVERSITY

MATH. SCIENCES RESEARCH INSTITUTE

NEW MEXICO STATE UNIV.

OREGON STATE UNIV.

\author{
STANFORD UNIVERSITY \\ UNIV. OF BRITISH COLUMBIA \\ UNIV. OF CALIFORNIA, BERKELEY \\ UNIV. OF CALIFORNIA, DAVIS \\ UNIV. OF CALIFORNIA, LOS ANGELES \\ UNIV. OF CALIFORNIA, RIVERSIDE \\ UNIV. OF CALIFORNIA, SAN DIEGO \\ UNIV. OF CALIF., SANTA BARBARA
}

\author{
Daryl Cooper \\ Department of Mathematics \\ University of California \\ Santa Barbara, CA 93106-3080 \\ cooper@math.ucsb.edu \\ Jiang-Hua Lu \\ Department of Mathematics \\ The University of Hong Kong \\ Pokfulam Rd., Hong Kong \\ jhlu@maths.hku.hk \\ Paul Yang \\ Department of Mathematics \\ Princeton University \\ Princeton NJ 08544-1000 \\ yang@math.princeton.edu
}

These supporting institutions contribute to the cost of publication of this Journal, but they are not owners or publishers and have no responsibility for its contents or policies.

See inside back cover or msp.org/pjm for submission instructions.

The subscription price for 2016 is US $\$ 440 /$ year for the electronic version, and $\$ 600 /$ year for print and electronic.

Subscriptions, requests for back issues and changes of subscribers address should be sent to Pacific Journal of Mathematics, P.O. Box 4163, Berkeley, CA 94704-0163, U.S.A. The Pacific Journal of Mathematics is indexed by Mathematical Reviews, Zentralblatt MATH, PASCAL CNRS Index, Referativnyi Zhurnal, Current Mathematical Publications and Web of Knowledge (Science Citation Index).

The Pacific Journal of Mathematics (ISSN 0030-8730) at the University of California, c/o Department of Mathematics, 798 Evans Hall \#3840, Berkeley, CA 94720-3840, is published twelve times a year. Periodical rate postage paid at Berkeley, CA 94704, and additional mailing offices. POSTMASTER: send address changes to Pacific Journal of Mathematics, P.O. Box 4163, Berkeley, CA 94704-0163.

PJM peer review and production are managed by EditFLOW ${ }^{\circledR}$ from Mathematical Sciences Publishers.

\section{PUBLISHED BY}

\section{mathematical sciences publishers \\ nonprofit scientific publishing}

http://msp.org/

(C) 2016 Mathematical Sciences Publishers 


\title{
PACIFIC JOURNAL OF MATHEMATICS
}

\author{
Volume $281 \quad$ No. $2 \quad$ April 2016
}

The Eisenstein elements of modular symbols for level product of two 257 distinct odd primes

DEBARGHA BANERJEE and SRILAKSHMI KRISHNAMOORTHY

Primitively generated Hall algebras

ARKADY BERENSTEIN and JACOB GREENSTEIN

Generalized splines on arbitrary graphs

Simcha Gilbert, JULiAnNA TyMOCZKO and SHIRA ViEL

Good traces for not necessarily simple dimension groups

DAVID HANDELMAN

On Fourier coefficients of certain residual representations of symplectic groups

DIHUA JIANG and BAIYING LIU

On the existence of central fans of capillary surfaces

AMMAR KHANFER

Surfaces of prescribed mean curvature $H(x, y, z)$ with one-to-one central projection onto a plane

FRIEDRICH SAUVIGNY 